\title{
Lapurdum
}

LAPURDUM Euskal ikerketen aldizkaria | Revue d'études basques |

Revista de estudios vascos | Basque studies review

2| 1997

Numéro II

\section{L'Aufklärung et la basquité}

\section{Pierre Bidart}

\section{OpenEdition \\ Journals}

\section{Édition électronique}

URL : http://journals.openedition.org/lapurdum/1828

DOI : 10.4000/lapurdum.1828

ISSN : 1965-0655

\section{Éditeur}

IKER

\section{Édition imprimée}

Date de publication : 1 octobre 1997

Pagination : 303-338

ISBN : 2-84127-142-0

ISSN : $1273-3830$

\section{Référence électronique}

Pierre Bidart, «L'Aufklärung et la basquité », Lapurdum [En ligne], 2 | 1997, mis en ligne le 01 septembre 2010, consulté le 30 janvier 2020. URL : http://journals.openedition.org/lapurdum/1828 ; DOI :

10.4000/lapurdum.1828 
Pierre BIDART

\section{L'AUFKLÄRUNG ET LA BASQUITÉ}

Si l'Aufklärung n'a pas inventé la basquité, grande a été pourtant sa contribution à sa structuration et à sa promotion au sein de l'Europe. Alors que la pensée philosophique et linguistique allemande décèle dès le XVIII' siècle l'intérêt de la basquité dans sa dimension linguistique, c'est au cours du XIX $X^{e}$ siècle, à la faveur d'une remarque de Jules Michelet dans son Histoire de France (1833-1846, 1855-1867) que la France découvre les travaux du linguiste allemand Wilhem Von Humboldt consacrés à la bascologie. Cet écart dans le temps ne fait que souligner les différences dans les conditions de formulation des objets scientifiques et leur statut, la complexité des représentations et des échanges culturels, voire les contentieux entre l'Allemagne et la France.

Dans un récent article intitulé "De l'Allemagne et de la France - Les faux semblants d'une opposition", Jean-Yves Guiomar souligne le poids immense et malheureux de ces oppositions stéréotypées entre une France terre des Lumières et de la Raison Universelle et une Allemagne empêtrée depuis le XVIII dans le Volksgeist, mais aussi entre une "bonne" Allemagne, avec Kant, Schiller, Goethe, et une "mauvaise" Allemagne, née avec Herder et triomphante avec Bismarck et Hitler, en passant aussi, comme beaucoup le pensent, par Hegel.

La canonisation de ces oppositions a emprunté différentes voies y compris celle des analyses d'historiens : "Pour les Français, une Nation est une communauté spirituelle (...), l'apparenance à un Etat repose sur une sorte de contrat. Cette conception relève donc du principe rationaliste du droit naturel, c'est par conséquent une conception à la fois individuelle et également universaliste comme la raison. Elle est liée par là au libéralisme dont elle devient une sorte de complément en matière de droit public international. Elle se rattache au principe de souveraineté du peuple”. Quant à la conception allemande, "elle s'est formée peu après en partant des théories du philosophe allemand Herder (...) Pour les Allemands, la nation est un organisme naturel doué d'un instinct vital, d'un génie propre" qui repose sur l'histoire et non sur la raison. En cela, la conception allemande est anti libérale. Jean-Yves Guiomar examine, entre autres, le domaine de la linguistique où les compétences supposées des deux pays sont nettement soulignées par la tradition scientifique alors que ces deux compétences trouvent en réalité une même filiation dans les travaux des linguistes français du XVIII' siècle. Ainsi, on a fait de l'Allemagne le pays de la grammaire comparée, la France ayant été identifiée comme le pays de la grammaire générale. Il est vrai que l'Allemagne a compté d'illustres représentants de la linguistique comparée avec Jacob Grimm (grammaire allemande, 1819 et 1822), Franz Bopp (langues indo-européennes, 1816 et 1833), Friedrich Diez (langues romanes, 1836) et Johan Kaspar Zeuss (langues cel tiques, 1853). Or, nous savons aujourd hui que ces travaux doivent leur origine aux débats suscités par L'Essai sur l'origine des connaissances humaines de Condillac (1746). Le Traité sur l'origine du langage de Herder (1772) se situe dans le prolongement de cet ouvrage. Aussi, cette opposition entre une linguistique rationaliste de 
caractère français et une linguistique historiciste de caractère allemand ne tient-elle pas debout. Les modes de développement inégalitaire de ces travaux s'expliquent notamment par l'histoire des institutions universitaires, l'université allemande ayant bénéficié, note J.Y. Guiomar, bien plus tôt que les universités françaises de chaires de linguistique comparée

L'effet le plus spectaculaire de l'Aufklärung se remarque dans le nombre de travaux consacrés à la langue basque et dont l'Allemagne contemporaine peut encore témoigner. La complexité de la structure linguistique du basque confortait heureusement la théorie des relations entre les propriétés d'une langue et le génie du peuple locuteur.

Parallèlement à cet engouement pour la bascologie tant magnifiée dans l'oeuvre de G. de Humboldt, la pensée de l'Aufklärung ne cessera d'inspirer directement ou indirectement, via la philosophie allemande, les interprètes de la basquité dans sa formulation nationaliste, en Pays Basque d'Espagne, à la fin du XIX' siecle. D'autres. marques très sensibles - étudiées plus loin - se remarquent dans les travaux de l'anthropologue Barandiaran et les textes littéraires de l'écrivain basque Mirande, fortement influencé par Spengler, lequel se revendique pour une part de la philosophie de Herder.

En France, les préoccupations empiriques de description des singularités provinciales qui animent le champ ethnographique en voie de constitution poussent une cohorte d'observateurs, au statut scientifique incertain, à s'intéresser à une société basque qui ne manque pas de "curiosités", et qui devient le point de convergence de trois axes géographiques: l'Allemagne, l'Angleterre avec Oxford, et Paris.

\section{Aufklärung et les prolégomènes anthropologiques de la basquité}

Face aux Lumières françaises pour lesquelles les activités spéculatives autour de la fonction linguistique n'occupaient pas une position centrale, l'Aufklärung se distingue par l'importance manifeste qu'elle accorde au fait linguistique, en l'associant à un large front de considérations philosophiques liées aux thèmes de la nation, de la culture de la religion, de l'histoire, de la nature, et initiant ainsi une véritable anthropologie de la société.

Une telle convergence de réflexions sur la question linguistique aboutira à l'automisation de la pensée linguistique et à la fondation de la "linguistique comparative" dont Guillaume de Humboldt sera le célèbre interprète. L'originalité de la contribution de l'Aufflärung ne peut se comprendre sans aborder les principes et les options philosophiques et religieuses qui expliquent son incarnation allemande.

La philosophie des Lumières, par l'hétérogénéité et les ambitions intellectuelles des questions qu'elle entendait traiter, contenait ou constituait une véritable anthropologie, mot nouveau désignant la matrice unitaire des savoirs du XVIII' siècle, ce que Michèle Duchet résume ainsi : "Science de l'homme, science du langage, étude des langues, genèse de l'entendement, histoire des sociétés, production littéraire, tout cela constitue pour les hommes des Lumières une seule et même activité et participe d'un même discours" ‘. Dégager les fondements culturels de l'anthropologie de l'Aufklärung revient à en examiner les orientations principales et les sens les plus significatifs, dans un contexte de concurrence culturelle entre la France et l'Allemagne et de divergence profonde quant à la manière de penser le rapport social à la religion et à la culture. 
La concurrence culturelle entre les deux pays trouve son origine dans le prestige aristocratique dont bénéficient la langue et la culture françaises, lesquelles s'imposent en tant qu incarnation de la modernité et outils raffinés d'expression du langage de la raison. Leur triomphe en Europe et au-delà marque l'aboutissement des promesses formulées sous la Renaissance tandis qu'elles symbolisent la nouvelle Grécité dont l'écho saisit toute l'Europe que Herder étudiera dans son Journal de mon voyage en l'an $1769^{\circ}$ et à laquelle G. de Humboldt sera également sensible. Le rayonnement des lettres françaises ne pouvait manquer d'irriter une conscience allemande tentée de se soustraire à leur domination. Exercice cependant difficile car on ne modifie pas si aisément les schémas culturels - en particulier les conceptions historiques - qui légitiment cette domination. Aussi la singularité allemande va-t-elle s'organiser, face à une francité triomphante, autour d'un concept mobilisateur, celui de la germanité. Cette entreprise de formalisation conceptuelle et de structuration de la singularité allemande passait par une récriture de l'histoire, une redéfinition des séquences historiques distinguant les civilisations conçues jusque-là selon la conception voltairienne qui voyait dans la Renaissance le moment historique essentiel de rupture avec l'obscurantisme médiéval. L'ouvrage de Herder, Une autre philosophie de l'histoire (1773), fort critique à l'égard de Voltaire, illustre très précisément cette ambition intellectuelle d'élaboration d'une version de l'histoire favorable désormais à la germanité qui doit trouver sa place dans le cours de l'histoire de l'humanité grâce en particulier au protestantisme. Il s'agit de procéder au réexamen général des relations entre l'histoire et le christianisme à partir de cette idée centrale de l'Aufklärung que la germanité s'incarne dans le protestantisme. Cet exercice simultané de formulation d'une germanité active et de positionnement historique de celle-ci sera accompli pour l'essentiel par Herder et par Fichte qui - trait propre à l'Aufklärung - se réferent sans cesse aux Lumières françaises pour s'en démarquer immédiatement. Les réajustements historiques engagés par ces deux auteurs s'appuient sur le rappel du sens de l'œuvre luthérienne qui prônait le retour au christianisme original face à un catholicisme confondu avec le règne de la superstition et de la corruption. La révolution luthérienne qui annonçait les temps modernes fut elle-même entravée par de multiples guerres, retardant la formation d un Etat national allemand alors que la monarchie catholique française édifiait un solide Etat-Nation. Se fondant ensuite sur le concept luthérien d'une historicité souterraine ${ }^{6}$ représentée par la Providence, force invisible qui anime le cours de l'histoire au même titre que les corpus sémantiques propres aux langues ellesmêmes, Herder et Fichte estiment que cette disparité est provisoire et déplacent les seuils et les symboles des ruptures historiques; à la Renaissance se substitue la Réforme ' qui détermine aujourd' hui le destin de la nation germanique. A la faveur de ce déplacement, les deux philosophes tracent une nouvelle voie de l'histoire : la Germanie se charge d'une dimension historique apte à revendiquer le rôle de héraut d'une authentique modernité, véritablement chrétienne. Cet emboîtement entre la germanité et le protestantisme, sur lequel insistera Hegel, deviendra l'argument nodal de la pensée politique et culturelle allemande.

Le postulat d'une germanité enchâssée dans le protestantisme s'opposait frontalement au modèle d'une francité articulée sur le discours anti-religieux des Lumières de même que la foi en une glorieuse germanité porteuse d'une authentique modernité heurtait la théorie culturelle de l'universalité de la langue et de la culture françaises et les hiérarchies linguistico-culturelles légitimées par celle-ci, à partir desquelles était déniée toute qualité aux langues et cultures non porteuses d'historicité, c'est-à-dire toutes celles qui représentent des traces de I'Ancien Régime et plus précisément le passé médiéval identifié aux temps de la barbarie. Si l'Aufklärung procède à la réha- 
bilitation du Moyen Age et de ses cuvres, les Lumières françaises préfèrent la Renaissance et sa rhétorique rationaliste naissante.

De son côté, Hegel ne manquera pas d'aborder certaines des conséquences de l'articulation entre la germanité et le protestantisme qui se fonde à ses yeux, sur le principe d'une affinité entre ces deux états. D'une part, l'attachement des Germains aux pratiques coutumières et simultanément leur méfiance - à cause de leur conscience d'appartenance à une communauté naturelle - à l'égard de toute structure politique centraliste organisée selon la théorie du droit universel (cette attitude ayant empêché un processus de modemisation étatique qui, par contre, a abouti en France). Par ailleurs, l'incitation du croyant allemand au sein du protestantisme à vivre sa foi et sa condition humaine dans le cadre de l'intériorité de sa conscience, d'où le rôle incontoumable de l'Eglise réformée dans le travail pédagogique de formation de la conscience allemande, au titre d'une suppléance face aux carences de l'institution politique.

Si le titre de l'ouvrage de Herder, Une autre philosophie de l'histoire pour une contribution à l'éducation de l'humanité. Contribution à beaucoup de contributions du siècle cache mal une intention polémique à l'égard surtout des "Lumières françaises" ". la pensée herdérienne ne manque pas pour autant d'originalité par les positions et les développements théoriques et empiriques qu'elle adopte. On peut souligner une première contribution dans l'affirmation d'une théorie du relativisme universel qu'il oppose aux philosophes défenseurs de la supériorité du XVIII' siècle et qu'il applique "dans son projet" de traiter des valeurs propres à chaque nation, à chaque siècle, "fleurs qui sous tel ciel poussent et prospèrent sans soin, et là meurent ou jaunissent misérablement" '. Si le thème du relativisme historique et ethnique avait déjà été abordé par Saint Evremond, l'abbé du Bos, Voltaire (Micromegas) et Montesquieu (Lettres persannes), Herder entend reconsidérer les schémas de périodisation de l'histoire et contester les classicismes et les orthodoxies tant en littérature et philosophie qu'ailleurs.

Pour qualifier les séquences de l'histoire, Herder fait appel au procédé métaphorique : il parle des âges de l'humanité, identifiant l'Orient biblique à l'enfance, la jeunesse à la civilisation égyptienne, l'adolescence au monde grec, la maturité et le déclin à la romanité ; il utilise également l'image de l'arbre dont les racines, le tronc, les branches, les feuilles sont illustrés par autant de nations singulières ${ }^{10}$. D'où une vision organiciste de la durée des civilisations et de leurs relations mutuelles commandée par les lois générales du vivant, avec une admiration insistante pour la période de l'enfance (de même d'ailleurs que pour les valeurs patriarcales), associée aux vertus de spontanéité de fraîcheur et d'authenticité, toutes qualités fondamentales qui déterminent, aux yeux de Herder, la valeur d'une culture ou d'une civilisation. Par deux fois, Herder invoque le temps de l'enfance dans ses schémas de périodisation de l'histoire : une fois pour qualifier l'enfance de l'humanité, symbolisée par la civilisation orientale, une autre fois pour désigner l'enfance de la Modernité, symbolisée par le Moyen Age dont il engage la réhabilitation. A chacun de ces âges, ses qualités ou ses défauts : au merveilleux de l'enfance font suite le désir d'entreprise et la grâce de l'adolescence, le sérieux et le réalisme de la maturité, la froide répétition et l'atrophie décadente de la sénescence" ".

Herder applique la méthode comparative tout à la fois diachroniquement - pour l'examen de la succession des civilisations orientale, égyptienne, grecque, romaine et moderne - et synchroniquement - dans l'étude des singularités françaises et alle- 
mandes. Parmi les auteurs les plus contestés figurent Voltaire, d'Holbach, Helvétius, Montesquieu à côté de Hume et d'autres. Ils sont d'ailleurs peu ou pas cités. Et à la faveur de ce comparatisme transhistorique et transculturel, Herder expose une philosophie de l'histoire, manifestement conservatrice dont, il modifiera, par la suite, les premières orientations.

Face à l'impuissance des mots à saisir la complexité et la singularité d'une nation, l'âme d'un peuple, "seul le créateur, dit-il, pense l'unité d'une nation et de toutes les nations dans toute leur diversité, sans que cela fasse disparaître à ses yeux l'unité" 12 . Vigoureusement dénoncé par les philosophes des Lumières françaises, le préjugé trouve grâce à ses yeux : "Je préjugé est bon, en son temps, car il rend heureux. Il ramene les peuples à leur centre, les rattache plus solidement à leur souche (...). La nation la plus ignorante, la plus remplie de préjugés est à cet égard souvent la première" ${ }^{13}$.

Il canonise la vertu "d'endurance des peuples du Nord" qu'il oppose à "la chaude serre aromatique de l'Est et du Sud" ${ }^{14}$. C'est aussi le Nord qui prit le relais de la romanité décadente, en apportant la "Nature" à la place des arts, "le bon sens nordique" à la place des sciences, "des moeurs fortes et bonnes bien que farouches" à la place du raffinement. C'est enfin là que s' affirma "l"esprit gothique", "chevalerie nordique au sens le plus large - grande manifestation de tant de siècles, de pays et de situations" ${ }^{16}$ - qui constitua l'ossature culturelle, politique et sociale et que rend compte une riche polysémie : "penchants paternels, et sainte vénération du sexe féminin, amour inextinguible de la liberté et du despotisme, religion et esprit guerrier, ordre strict, solennité et goût étrange de l'aventure" ${ }^{17}$.

S'en prenant une nouvelle fois aux Voltaire, Hume, Richardson et Iselin qui raillent la société médiévale, il procède à la réhabilitation des temps médiévaux sans pour autant en faire l'éloge (car "tout ceci est vrai et n'est pas vrai" ${ }^{18}$, dit-il, en parlant des travaux des précédents). Il défend ainsi, tout à tour, "les rudes résidences campagnardes (qui) empêchaient l'accroissement exubérant et malsain des villes, ces groupes dévorants des forces vitales humaines" 19 ; l'harmonie sociale entre la lignée et les familles, le maître et le serviteur, le sujet et le roi ${ }^{2 n}$, tandis que "le manque de commerce et de raffinement empêche le relâchement des moeurs". Il se félicite même de l'existence de la barbarie: "si le ciel" ne vous avait pas fait précéder par les temps barbares et ne les avait maintenus si longtemps parmi tant d'à-coups et de heurts pauvre Europe policée, qui dévores ou relègues tes enfants, que serais-tu avec ta sagesse ? - Un désert !" :?. Il dénonce l'idée que la morale laïque - représentée par "l"art de la législation" pût suffire à "cultiver les nations" $"$, idée présente chez Helvétius (De l'Esprit, 1769), d’Holbach (Sistème de la Nature, 1770) et De Chastellux (De la félicité publique, 1772).

Il stigmatise l'action et le succès des académies et des salles d'art et l'importance reconnue à l'éducation ${ }^{25}$. Il préferre la clôture des mentalités dans des espaces restreints, "la chaumière patriarcale" et "la petite république d'hommes" ${ }^{26}$, la nation fermée sur elle-même, le temps où "tout était si étroitement naturel" ${ }^{27}$, les contrées restées "sauvages et barbares" à l'instar de l'Irlande ("c'est une honte pour l'Angleterre que 1'Irlande soit si longtemps restée sauvage et barbare : elle est policée et heureuse" ${ }^{28}$ ). L'effacement des caractères naturels entraîne la disparition des caractères nationaux. du sentiment national et facilite ainsi le déclin. Et le pire des destins pour un peuple n'est-il pas de perdre sa singularité nationale ; "on ne peut causer de plus grand dommage à une nation qu'en la dépouillant de son caractère national, de ce qu'il a de spé- 
cifique dans son esprit et sa langue" ${ }^{29}$. La rationalité domesticatrice à l'oeuvre dans les jardins "à la française" lui apparaît comme un contre-sens intellectuel : "on inventa (...) une architecture sur le plan même et avec toutes les productions de la nature - les jardins ! Plein de proportions et de symétries ! Plein d'éternelles jouissances et nature toute nouvelle sans nature !" 30 .

En somme, il s'insurge contre la prétendue supériorité du XVIII siècle, tant magnifiée par Voltaire dans son Siècle de Louis XIV, ce qu'il assimile à la sénilité (lui qui reproche tant aux "philosophes des Lumières" de résumer une époque par un mot succombe à cette même tentation!). Et la réhabilitation du Moyen Age le conduit à l'arrimer à la période contemporaine pour les besoins du moment, c'est-à-dire la constitution et l'éloge de la germanité. Cette appréhension favorable des temps médiévaux, caractéristique du Sturm und Drang et du romantisme, diffêre néanmoins de la célébration catholique du Moyen Age operée par des auteurs comme Novalis dans La Chrétienté ou l'Europe (1799). L'état de félicité dont il fait la finalité de toute vraie civilisation, il le remarque surtout dans l'âge de I'enfance qui est aussi le temps des patriarches : "Etre humain, homme, femme, père, mère, fils héritier, prêtre de Dieu, régent et père de famille, c'est par tous les millénaires qu'il devait alors être forme ! Et éternellement, en dehors du Règne de mille ans et des fables des poètes, éternellement le pays des Patriarches et la tente des Patriarches demeurera l'Age d'or de "l'humanité enfant" " ${ }^{3}$. Temps fécond que celui de l'enfance, des temps primitifs, par leurs talents poétiques, alors que l'époque modeme - celui de la prose - symbolise le déclin, cette idée inspirera les écrits sur De l'effet de la poésie surles moeurs dans l'Antiquité et de nos jours (1778) ${ }^{32}$. Cette ferveur pour l'activité poétique le poussera à croire en l'authenticité des poèmes épiques d'Ossian, comme il le dit dans Du Naturel et de l'an allemand. Extraits d'une correspondance sur Ossian et des chants des anciens peuples (1773). Il souhaite dans ses Fragments (1768-1769) le renouveau de la poésie allemande par la substitution de la mythologie germanique à la mythologie gréco-latine. Il procède à une vaste enquête sur l'âme allemande à travers ses poésies, ses contes et ses légendes matérialisée dans son recueil de Chants populaires (1773). Bien des approches romantiques similaires seront inspirées par la conception herdérienne du chant populaire, qu'il s'agisse de recueil de chansons (Des Knaben Wunderhom de Brentano, 1805), ou des contes populaires (Teutsche Volksbücher de Görres, en 1807 ; Hansmärchen des Frères Grimm, 1812 ; Deutsche Sagen de Wilhelm Grimm, 18161818) ${ }^{3 ?}$

En tant que théoricien de la relativité historique, il estime que toutes les civilisations connaissent inexorablement les mêmes phases de progrès et de déclin; comme théoricien de la relativité ethnique ${ }^{4}$, il affirme que toutes les nations se correspondent dans une égale nécessité, une égale originalité, un égal mérite, un égal bonheur "

Les fondements du principe des nationalités sont ainsi établis. Aussi, l'histoire des civilisations ne se construit-elle pas autour du concept de progrès (comme l'entendent les Lumières) mais plutôt à partir du concept de continuité ("Fortgang"), non pas une continutité qui signifie une répétition ("En aucun pays, dit-il, la culture n’a pu revenir en arrière, et redevenir une nouvelle fois ce qu'elle avait été" ${ }^{-6}$ ) mais une continuité faite de discontinuité (chaque culture emprunte auprès de la précédente). Et la prospection historique sur les cultures populaires ${ }^{37}$ vise à mettre en exergue "l' esprit du peuple", ou encore "I'esprit général" sous la forme du Nationalgeist. Herder préfere en effet l"histoire du peuple à celle des dynasties ou des armées; il pense à la possibilité de la reconstruction anthropologique d"un peuple à partir de fragments disponibles. Il conteste le primat de la raison et son arrogance à vouloir déterminer le cours 
de l'histoire (tout en ne cessant pas d'utiliser certains aspects du rationalisme) à laquelle il oppose l'histoire, de manière à fonder une autre philosophie de l'histoire. II valorise ces individus collectifs que sont les peuples au détriment des universaux, ces peuples qui sont des vrais dépositaires de la culture nationale et dont les qualités morales se remarquent dans la ferveur et la densité de son état naturel que singularisent le sens de la hiérarchie, les valeurs religieuses et les rapports sociaux harmonieux. Cet état naturel se distingue aussi pour sa spontanéité, ses facultés créatrices présentes au sein du langage poétique dans lequel excelle le peuple. Ces postulats, Herder les accompagne de considérations de nature vitaliste, remarquables dans une syntaxe récurrente ("Énergie vitale", forces vitales", etc.), dans ses métaphores de la civilisation/arbre ou de la nation/organisme. En réalité, son œuvre est construite autour de trois idées ${ }^{38}$ divergentes parfois jusqu'à la contradiction (qui, par ailleurs, s'opposent souvent à l'Aufklärung ${ }^{39}$ tout en lui empruntant des arguments explicatifs) : l'affirmation de l'universalisme chrétien dont les retombées culturelles, inestimables, sont à l'origine de la modernité, l'absolutisation des qualités culturelles d'une race ou d'une nation singulière par essence (qu' accompagne Herder du refus de l'imitation. ce qui conduit à l'autarcie culturelle), la proclamation d'une relativité généralisée induisant le refus de toute absolutisation culturelle. C'est à partir de cet entrelacement philosophique que s'exercera son influence, que l'on imaginera des filiations (réelles ou fictives), que lui sera réserveé une postérité souvent peu flatteuse. Que d'analyses sous-entendant ou évoquant explicitement une filiation entre le IIIe Reich et la pensée de Herder ${ }^{\text {sn }}$ ! L'hétérogénéité des concepts et des paradigmes utilisés par Herder (nature, culture, modernité, peuple, "esprit national", nation, primitivisme. etc.) de même que la diversité des relations logiques entre ces concepts et ces paradigmes laissent imaginer la nature des influences et des détournements possibles. Tandis que l'universalisme abstrait prôné par la philosophie des Lumières contient l'idée que la nation est à la fois le produit et la vectrice de la Modernité, l'Europe se conforte dans un ethnocentrisme culturel troublé par les relations critiques entre cultures dominantes. Aux tensions entre celles-ci - illustrées en particulier par les exemples français et allemand - s'ajoutent d'autres tensions nouvelles entre cultures dominantes et cultures non officielles telles qu'on peut les observer en Espagne où le débat sur les rôles respectifs de l'hispanité et de la basquité prend une tournure conflictuelle avec les écrits, du côté basque, d'hommes d'Eglise, Larramendi, Astarloa, Moguel, entre autres. L'influence de Herder est manifeste auprès de Moguel lorsque celui-ci oppose le basque rustique, Peru Abarca au barbier culte Maisu Joan ". Moguel et Astarloa seront les correspondants d'Humboldt lorsqu'il entreprendra son voyage en Espagne.

Les oppositions binaires établies par Moguel, - le rustique/le culte, bon bascophone/mauvais bascophone, etc. - renvoient aux oppositions binaires formulées par Herder dans son Traité sur l'origine des Langues où il s'en prend aux "froids philosophes" vivant dans les salons. Elles peuvent être, selon W. Moser, construites ainsi : l'homme grossier de la nature/le philosophe de salon, indivision organique/démembrement analytique, expérience-sentiment/raison-intellect, naturel/artificiel, vie-vivacité/mor, principe d'activité indivis/activité disséquante, chaleur-énergie/froid, principe liquide-source/aridité-assèchement ${ }^{42}$. Par contre, la mission du bon philosophe s"énonce dans l'analyse des formes du primitif : "d'une part le philosophe entend maintenir et respecter la spécificité des temps primitifs, des moments de genèse, il désire se donner leur esprit, son travail est donc porté par l'élan qui transpose le sujet dans I'objet. D' autre part, il lui faut aussi faire progresser les connaissances humaines en rapportant du butin de son voyage cognitif au pays du primitif (inutile de rappeler 
que cette métaphore se pratiquait beaucoup au XVIII siècle !). Herder écrivait à la fois en philosophe nostalgique et en philosophe éclairant" ".

Les développements de Herder sur le thème populaire visent moins à poser les fondements d'une science du populaire, telle que celle-ci sera désignée au XIX' siècle sous le terme de Volkskunde qu'à définir une esthétique du populaire, ce que H. Bausinger traduit ainsi : "Tout cela met en évidence que la poésie populaire n'est pas traitée comme un fait de tradition orale, mais comme fiction créative faisant coincider "populaire" et "artistique" 4.

En faisant du peuple le contre-point a-historique du concept de classe sociale, le "lieu" d'incarnation de "l'esprit national" et de l'esthétique, la pensée herdérienne sera à l'origine d'un mouvement culturel (d'inspiration aristocratique-bourgeoise), né vers le milieu du XIX ${ }^{c}$ siècle recherchant la mise en scène des aspects festifs de la société basque (danses, pelote, improvisation, concours de poésie). Les références constantes au charme des hommes et des femmes basques, la propension des observateurs à faire l'inventaire des "curiosités" du Pays Basque contribuant, comme on le verra plus loin, à illustrer la place d'une approche esthétisante du "populaire" basque perçu dans sa strate paysanne.

Enfin, c'est par ses Idées pour la philosophie de l'histoire de l'humanité que Herder a pu certainement intéresser Humboldt à l'étude des expressions culturelles primitives et en particulier à celle observable en Pays Basque, laquelle lui inspire les réflexions suivantes: "Les peuples mêmes qui sont très connus sous le nom de Basques et de Cantabres se sont montrés dans l'histoire ancienne un peuple rapide, léger, vaillant, aimant la liberté. Ils accompagnèrent Annibal en Italie et leur nom chez les poètes romains est redoutable : ce sont eux, ainsi que les Celtes d'Espagne qui rendirent le plus difficile aux Romains l'asservissement de ce pays, en sorte qu'Auguste fut le premier à triompher d'eux, et peut-être seulement en apparence : car tous ceux qui ne voulurent pas être asservis se retirèrent dans les montagnes. Lorsque les vandales, les Alains, les Suèves, les boths et autres peuples teutoniques opérèrent leur farouche traversée des Pyrénées et que quelques uns fondèrent des royaumes dans leur voisinage ; ils étaient encore le peuple vaillant et remuant qui $n$ avait pas perdu courage sous les Romains, et quand Charlemagne revenant de vaincre les Sarrasins d'Espagne traversa leur pays, ce furent encore eux qui, au moyen d'une attaque par ruse, provoquèrent cette défaite de Roncevaux, si célèbre dans les anciens romans, où périt le grand Roland. Plus tard, ils donnèrent en Espagne et en Aquitaine du fil à retordre aux Francs. Comme auparavant aux Suèves et aux Goths ; également lors de la reconquête du pays sur les Sarrasins, ils ne restèrent pas inactifs et même conservèrent leur caractère jusque dans les siècles de la plus profonde oppression barbare par les moines. Lorsqu'après la longue nuit, une aurore de la science se leva pour l'Europe, elle commença à poindre grâce à la joyeuse poésie des Provencaux dans leur voisinage, en partie dans ces pays habités par ceux qui plus tard donnèrent aussi à la France nombre d'esprits joyeux et éclairés. Il serait souhaitable que nous connaissions mieux la langue, les moeurs et l'histoire de ce peuple rapide et joyeux et que, tel Mac Pherson parmi les Gaëls, un second Larramendi se mette parmi eux en quête des restes de leur vieil esprit national basque $(. . .)^{+5}$.

Par ces quelques remarques ayant valeur d'un programme, Herder fonde ce qui devait devenir Ia tradition bascologique allemande. 


\section{II - Langue et peuple dans l'Aufklärung}

L'anthropologie des Lumières s'est fortement appuyée sur des considérations linguistiques ${ }^{\text {th }}$ dont les centres d'intérêt différaient sensiblement selon que l'on était en France ou en Allemagne, même si la linguistique allemande réagissait souvent aux assertions de la linguistique française.

Du côté français, les débats portaient notamment sur l'origine du langage articulé, la langue des bêtes, la faculté de communication, l'ordre naturel des mots ; ce dernier élément, justifié par la théorie rationaliste et appliqué au français, devait servir à faire l'apologie des qualités du français ${ }^{47}$ contestée par la théorie sensualiste ${ }^{48}$ [représentée officiellement par Condillac] qui, elle, entendait insister sur la liberté dans l'ordre des mots - liberté à laquelle faisaient écho les libertés politiques nouvelles nées de la Révolution Française (selon les formules consacrées, le grammairien-philosophe gagnait le nouveau titre de grammairien-patriote) -, la théorie de l'ordre naturel des mots sera détoumée par les représentants de la pensée conservatrice (celle de la Restauration) contre la Révolution Française, observe U. Ricken ${ }^{49}$, en parlant de L. de Bonald. Et les questionnements linguistiques privilégient, bien entendu, le français (à travers le principe de l'universalité de la langue française), puis, selon les cas, l'anglais, l'italien, le latin et le grec ; les autres langues apparaissent peu dans les débats ; et sont exclus, cela va de soi, les ensembles linguistiques internes à la France, de par leur statut infériorisé de patois qui les soustrait de la modernité et même parfois de toute objectivation scientifique.

Du côté allemand, le souci de la fondation d'une germanité liée à l'affirmation culturelle de l'allemand ne gène nullement l'attention à d'autres langues que l'on aborde à travers la méthode comparative.

\section{A - De Herder à Fichte : les instrumentalisations de la langue.}

Ami. dans un premier temps, de Kant, Herder conçoit sa Métacritique de la "critique de la raison pure" (1799) comme un essai d'invalidation du discours de Kant sur les distinctions dans l'acte de connaissance entre l'être et le connaître. Le prétexte lui est fourni par le concours lancé par l'Académie de Berlin, en 1772, auquel il répond par son Üher den Ursprung der Sprache ("Sur l'origine du langage"). La critique à l'égard de Kant, accusé d'avoir produit une langue scolastique est vive : "celui qui a rendu artificielle la langue d'une nation (quelle que soit par ailleurs l'astuce qu 'il déploie) a corrompu l'instrument de la raison et le lui rend odieux. (...) La métacritique relève donc du protestantisme. Elle proteste contre tout papisme imposant ses règlements de façon non critique et non philosophique à la raison et à la langue" $s$.

Pour Herder, si la langue est "l'instrument de la raison", elle forme aussi le substrat du Gemeinsinn, c'est-à-dire du sens communautaire qui fonde la nation, celui-ci se distinguant du Gemeinsinn de la Popular philosophie entendue comme bon sens partagé par tous et éclairé par la raison ${ }^{51}$. C'est ce Gemeinsinn"2 qui légitime les jugements esthétiques, c'est-à-dire définit les critères du Beau et du goût. Et c'est l'articulation entre langue et Gemeinsinn que le IIl ${ }^{`}$ Reich s’empressera d'exploiter selon ses vues nationalistes. C'est aussi cette articulation que défend explicitement le mouvement nationaliste basque lors de sa formation à la fin du $\mathrm{XIX}^{\mathrm{c}}$ siecle dans les provinces basques d'Espagne et que l'on peut encore fréquemment noter dans les écrits contemporains ${ }^{53}$.

Le Révérend Herder n'est pas seul, bien entendu, à se préoccuper du devenir de la germanité. Un membre éminent de l'Aufklärung et de surcroît franc-maçon J.G. Fichte: 
manifeste dans un premier temps son admiration pour la Révolution Française qu“il défend ${ }^{\$ 4}$ contre des critiques anglaises " et allemandes ${ }^{\$ 6}$, puis intellectuellement meurtri par la fin de la Révolution et par les guerres napoléoniennes, il élabore de manière autre mais complémentaire une philosophie de la germanité qu'il expose dans son Discours à la Nation allemande ${ }^{57}$. Son enthousiasme pour la Révolution Française, Fichte le justifie par la contribution de cette dernière a l'invention d'une nouvelle phiIosophie du Droit, selon laquelle les peuples ont autorité pour changer les structures politiques et opérer ainsi ce que l' on appelle une "révolution", ce qui lui permet d'envisager, par effet d'entraînement, une transformation de la germanité en vecteur de modernité. La Révolution Française expérimente en réalité une sorte de mouvement historique circulaire né dans les forêts de Germanie" $s$, dotée jadis d'institutions de qualité. Dénaturé au gré des vicissitudes historiques ${ }^{*}$, cet idéal de germanité peut être réactivé à la faveur de la Révolution Française pour revenir à la surface de la conscience allemande. La perception d'un nouveau destin possible pour cet idéal de germanité le conduit naturellement à souligner la profondeur historique et les propriétés ontologiques de la langue allemande.

Malheureusement, l'ensemble des événements post-révolutionnaires provoquent l'amertume de Fichte qui se voit contraint de récuser ses premières analyses et convictions. La déception est à la mesure de la grandeur de sa foi en la Révolution Française et se convertit en sentiment de trahison à l'égard des valeurs et des symboles incamés dans la nation allemande. Son Discours à la nation allemande contient un véritable projet éducatif qui doit permettre la transformation de la nation allemande en sujet politique, culturel et moral [le thème de la formation du "moi", central dans l' oeuvre de Fichte, étant exposé explicitement dans la Doctrine de la science]. A défaut d'EtatNation institué, c'est la langue qui se trouve investie de la fonction de représentation symbolique de la nation allemande dont elle est aussi l" infrastructure culturelle essentielle.

Construit autour de quatorze discours, Le Discours à la nation allemande insiste avec force sur la capacité pédagogique de la langue allemande à l'égard de l'histoire, pour aboutir à une approche essentialiste de la germanité définissable ainsi : la germanité est parce que la langue allemande est. Le discours IV comporte plusieurs développements consacrés à la question linguistique à travers la distinction opérée entre l'allemand, langue vivante et les langues néo-latines, classées langues mortes, et soulignant la fonction heuristique possible des langues vivantes ${ }^{50}$. Et aux divisions sociales qui caractérisent une France partagée entre une classe cultivée formée par l'étude, maîtrisant la littérature et une classe populaire vivant de ses propres ressources, instinctuelle, inculte et étrangère à l'art, Fichte oppose une germanité réunissant en son sein, la raison et le sentiment, l'âme du peuple et les oeuvres d'ar ${ }^{(1)}$.

\section{b - Humboldt : le fait basque.}

Le travail de désacralisation philosophique et philologique de la langue (comme incarnation du Verbe) et la déconstruction des paradigmes linguistiques sur le plan du sens (on se réfere en particulier à la position privilégiée de l'hébreu) vont connaître avec $\mathrm{G}$. de Humboldt une étape décisive conduisant à la formation d'une science nouvelle. la linguistique et plus précisément "la recherche linguistique comparative, qui de par sa nature même, doit rester sur le plan des faits" ${ }^{62}$. Si Humboldt partage bien des analyses effectuées par Herder et par Fichte, il s'en éloigne dès le moment où il veut soustraire la question linguistique des considérations exclusivement idéologiques et en faire un objet d'analyse scientifique. 
A lire l'ouvrage de G. Raulet sur Aufklärung. Les lumières Allemandes, cité à plusieurs reprises ci-dessous, on ne peut que regretter de voir l'œuvre de Humboldt citée une seule fois avec son Essai sur les limites des compétences de l'Etat (1792), ouvrage qui est loin, à notre avis, de traduire la richesse et la nouveauté des apports de Humboldt. Il convient par contre de souligner l'intérêt de l'importante recherche de Jean Quillien sur l'Anthropologie philosophique de G. de Humboldt ${ }^{63}$, qui a par ailleurs mis en évidence la qualité des réflexions historiques de ce penseur ${ }^{64}$, dans son ambition de se distancier d'une part du dogmatisme philosophique et d'autre part, de l'empirisme étroit qui se condamne à l'accumulation des faits et à l'impasse.

L'histoire a traité de manière bien inégale les Frères Von Humboldt accordant ses faveurs à Alexander ${ }^{65}$ (1769-1859), l'explorateur, le climatologue, le géographe, l'archéologue, l'anthropologue, l'ami de Laplace, de Monge et de Gay-Lussac, au détriment de Wilhelm (ou Guillaume, 1767-1835), l'homme de lettres, l'esthète, le traducteur de Pindare et d'Eschyie, le philosophe, le linguiste et l'homme d'Etat ${ }^{\star}$. Des parcours presque similaires en bien des points (même éducation "éclairée", fréquentation des mêmes salons, mêmes amis dont Goethe) peuvent probablement expliquer le fait que la postérité ait pu les confondre souvent. Situation bien injuste et regrettable quand on sait son influence sur les travaux de Dilthey, Cassirer, Croce ou Chomsky. Il est vrai également que seule une partie minime de son cuvre a été traduite en français ${ }^{67}$.

Né dans une famille très aisée, d' ascendance prussienne par le père et franco-écossaise par la mère, G. de Humboldt bénéficie d'une éducation fortement imprégnée de l'esprit de l'Aufklärung. Sa formation universitaire achevée à Gottingen (qui deviendra le centre de la linguistique comparée), il vient à Paris, en 1789, "voir la Révolution" dont le spectacle l'éblouit. Il fréquente à Berlin, capitale intellectuelle de l'Allemagne et de l'Aufklärung à la fin du XVIII' siècle, les salons où l'on peut croiser l'élite intellectuelle, c'est-à-dire les salons de Nicolai, des Mendelssohn el des Herz. Cela lui permet de devenir le familier de Goethe, d'avoir pour amis Schiller. Fichte, Lesage, Forster, Jacobi et bien d'autres. En 1797, il s'installe à Paris pour quatre ans, en profite pour passer presque une année en Espagne et au Pays Basque. ce qui lui permet d'écrire en 1801 ses Fragmente der Monografie über die Basken. A partir de là, son intérêt pour les langues ne cessera de s'élargir, poussant ses investigations vers le chinois, le japonais, le sanscrit, les langues sémétiques et amérindiennes, entre autres, avec le souci constant d'appliquer une méthode pluridisciplinaire (le recours simultané à la philosophie, à la linguistique et à l'histoire) qu'il expose dans une correspondance adressée à Schiller, en 1802 : “Vous savez que je m'occupe depuis assez longtemps, de la manière la plus sérieuse, de l'étude du langage (Sprachstudium). Je crois avoir fait, en ce qui concerne les idées générales sur la question, des progrès non négligeables, $\mathrm{j}$ ai un plan général que $\mathrm{j}$ 'achèverai et présenterai, dès que j'aurai simplement tout d'abord des loisirs plus tranquilles. Ensuite. il faudra passer à sa réalisation, pièce par pièce, par moi et par d'autres, car mon plan vise à une encyclopédie générale de l'étude du langage dans son ensemble et par conséquent, de toutes les langues (...). Je relie toujours (cette recherche) à la philosophie et à l'étude des peuples" ${ }^{88}$. Cette démarche ${ }^{69}$ pluridisciplinaire repose sur la collaboration constante entre la réflexion théorique et la recherche empirique. entre la métaphysique et le positivisme. Parmi les questions qui inspirent la linguistique comparative naissante, figure celle de l'étude comparée des langues modernes et des langues anciennes, sujet qui justifie ses études sưr le basque ; "C"est un phénomène singulier que, parmi les essais scientifiques variés dont peut se prévaloir notre époque. 
un sujet de méditation et de recherche riche, important et d'intérêt général, soit resté totalement en friche - la comparaison des différentes langues anciennes et modemes" 7 .

Par ailleurs, la recherche linguistique ne vise pas seulement à la seule accumulation de connaissances sur un nombre de langues aussi grand que possible, elle est animée aussi par des considérations morales relatives à l'usage social de ces connaissances ; "la recherche linguistique comparative ne peut prétendre ouvrir des perspectives importantes et incontestables sur le langage, le développement des peuples et la promotion de l'humanité, qu'en se constituant en discipline autonome, ayant en elle-même son utilité et sa fin" " ${ }^{7}$. L'apport fondamental de Humboldt se trouve dans cette conception de la langue non pas comme une substance ou un objet achevé mais comme un mouvement continu, une activité, une énergie. ll établit une relation si étroite entre la langue prise comme un tout organique avec sa structure singulière, entendue comme une "œuvre d'art" élaborée par ses utilisateurs et la mentalité d'un peuple, qu'il lui paraît possible de déduire l'une ou l'autre, de lire dans la langue nationale l'esprit d'un peuple, la psychologie d'une nation. Aussi, l'individualité d'une nation - présente dans l'individualité d'une langue - doit-elle être préservée coûte que coûte (d'où la position centrale de la notion de continuité dans l'oeuvre de Humboldt, la rupture dans la conscience du passé étant la pire des choses imaginables dans l'hsistoire d'une nation). La passion de Humboldt pour l'étude des langues dans leur diversité se double de la passion de la défense de cette diversité linguistique, conformément aux principes scientifiques et moraux qui fondent sa théorie linguistique. La diversité linguistique et nationale renvoie aux modalités pratiques de segmentation et de réunion des membres de l'espèce humaine et permet d'imaginer le rassemblement de toutes ces nations dans le cadre d'une "alliance". La présence d'une téléologie politique et culturelle au sein de la philosophie de l'histoire de Humboldt correspond, à bien des égards, à la sensibilité religieuse et protestante (de même que pour Herder et Fichte) qui laisse entrevoir cette quête d'une fraternité universelle. Cette attitude intellectuelle interdit logiquement toute idée d'hiérarchisation culturelle des langues : chacune, de par son existence et son individualité, mérite considération ; chacun institue la médiation (s'institue en médiatrice) entre la part intérieure et la part extérieure, entre la subjectivité et l'objectivité. Le génie et la valeur des langues naissent de cette capacité que toutes partagent. "Le langage a été découvert comme la médiation entre le sensible et l'intelligible, l'homme et le monde puis à l'intérieur de l'univers langagier, second univers que l'homme interpose entre lui et le monde extérieur, la diversité des langues est la médiation entre l'Universalité de l'esprit humain et le singulier de chaque langue et en elle, de chaque individu parlant. La parole vivante, énergia, productrice continuée de sens, est l'ultime médiatrice entre l'individu dans l'instant et le tout des individus, passés, présents et à venir, entre l'atome concret, seule réalité, et l'humanitê" "n , résume excellemment J. Quillien.

\section{a) La langue basque ou le modèle du primitif}

Nous devons à Jules Michelet d'avoir attiré l'attention dans son Histoire de France $e^{73}$, sur les Recherches sur les habitants primitifs de l'Espagne à l'aide de la langue basque de Humboldt, tout en déplorant que la traduction de cet "admirable petit livre" ne fût pas encore réalisée. Elle le sera en 1866, par M. Marrast ${ }^{\prime 4}$, procureur impérial à Oloron Sainte Marie, dans le département des Pyrénées Atlantiques.

Lors de son séjour à Paris, Humboldı découvre avec émerveillement. à la Bibliothèque Nationale, le dictionnaire manuscrit de $\mathrm{S}$. Pouvreau et les Proverbes d'Oyhénart. Et, au cours de son voyage en Espagne, en 1800, il visite le Pays Basque, 
se faisant géographe, ethnographe et se passionnant pour une langue basque en qui il décèle les plus étonnantes qualités expressives de la pensée humaine. Il s'attire l'amitié du curé de Durango, Don Pedro Astarloa, dont les recherches lui servent de guide précieux. Il rencontre aussi des savants espagnols impliqués dans les débats linguistiques qui agitent l'Espagne d'alors, à savoir la critique de la place du basque dans le passé linguistique de la péninsule ibérique. Il fait également la connaissance du curé de Marquina, Don Antonio Moguel y Urquiza qui note à propos de Humboldt : "Un noble y sabio Prusiano, Llamado Varon de Humboldt se dedico in Paris a la lectura del arte y diccionario vascongados (...) Venia Comisionado de alguna sociedad de sabios que intentan descubrir las lenguas matrices primitivas y originales. Va a pubicar en breve, alguna obra sobre este idioma despreciado por muchos sabios españoles que debian confundirse en semejante exemple : el sabio Prusiano quere demontrar que el idioma bascongado es eloquente, puro y fecondo" " De manière intentionnelle certainement et avec habileté, le curé Astorloa inscrit les recherches de Humboldt dans la contre-offensive menée par les apologistes de la langue basque contre tous les détracteurs : "Aux yeux du bon curé, son suffrage vengeait bien l'euscara des dédains ridicules de certains auteurs espagnols qui, n’en comprenant point le mécanisme, le traitaient de jargon barbare et sans règles (V. Mariana)" ${ }^{76}$.

A son retour en Allemagne, Humboldt - qui souhaite consigner toutes ses observations et ses remarques dans un ouvrage d'ensemble - présente son projet dans un article inséré dans le Museum allemand de Frédéric Schlegel, avec pour titre : "Ankundigung einer Schrift über die baskische Sprache und Nation. nebst angabe des Gesichtpuncktes und Erhaltes desselben" ("Annonce d'un ouvrage sur la langue basque, et sur la nation basque étudiée d'après les données foumies par sa langue”). Humboldt résume ainsi les séquences thématiques de cet ouvrage. Dans la première, il compte, sous la forme d'une "relation de voyage", peindre "le sol, les moeurs et les caractères de cette race vive et intelligente, disséminée dans les vallées pyrénéennes, et sur la Côte de l'Océan, à la fois montagnarde et maritime, constituée depuis une haute antiquité en groupes indépendants unis par un lien fédératif, à la manière des petits peuples de la Grèce antique". Dans la seconde, consacrée aux développements linguistiques, l'auteur entend étudier le basque de manière diachronique, mais aussi par rapport aux autres langues en perspective d"un ambitieux projet scientifique appelé à être un monument de linguistique comparative : "Dans cet examen, je ne perdrai pas de vue les autres langues, car, pour moi, la connaissance du basque n'est qu'un des éléments de l'étude comparative qui permettra d'édifier un jour l'Encyclopédie générale du langage. Je comparerai le basque avec les autres langues, et j'indiquerai (si cela est possible) à quelle classe et à quelle famille il se rattache". Quant à la demière séquence, elle rappellera les finalités politiques qui accompagnent une telle entreprise philosophique, à savoir l'éloge de la diversité linguistique. Le positivisme trouve ainsi une légitimité et son sens dans une téléologie d'essence politique : la germanité avec l'Aufklärung, et ici, la promotion de la nation basque.

Les Recherches sur les habitants primitifs de l'Espagne s'ouvrent par une déclaration de méthode et d'intention. La toponymie, en fixant l'histoire, constitue une ressource incomparable pour la reconstitution de l'histoire d'un peuple : "Dans ces monuments [les noms de lieux] les plus anciens et les plus durables de tous, une nation disparue depuis longtemps nous raconte son histoire, il ne s'agit que de la comprendre" 77. Et l'ensemble de ces recherches doit permettre " $d$ 'asseoir sur de nouvelles bases l'histoire d'un pays et d'un peuple, et de nous fixer enfin sur l'origine, la propagation et les analogies de la langue basque, questions jusqu'ici si controversées" 7 . 
Humboldt apprécie nombre d'observations d'Astarloa mais conteste son adhésion à la théorie de Davies, théorie selon laquelle les purs mots celtiques les plus longs peuvent se ramener à des radicaux qui ne désignent pas les objets réels - la terre, l'eau, l'arbre - mais expriment seulement les différentes manières d'être et d'agir. Rétif à toute idée d'arbitraire, Astarloa voit dans le sens une conformité aux sons articulés par I'homme, aux bruits de la nature. Ainsi $\mathrm{O}$ désigne ce qui est rond ; $\mathrm{i}$, ce qui est aigu, tranchant ; U ce qui est creux, etc. ${ }^{79}$. Humboldt accompagne cette analyse naive par une autre : "il explique, [dit-il], gravement l'a de aarra ("homme") et l'e de emea ("femme") en disant qu'à son premier cri, l'enfant mâle fait entendre un $a$ et l'enfant

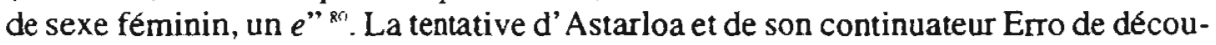
viir dans le basque "la langue mère de la race humaine" ${ }^{81}$ restera une vaine entreprise : “Tant que les linguistes basques $n$ ' auront pas renoncé à cette tentative universellement reconnue chimérique pour se borner à foumir leur part d'observations sur leur idiome, leurs travaux ne seront jamais d'une pleine utilité pour leurs compatriotes ni pour les étrangers" "2:. Etablir les relations de continuité entre les anciens noms de lieux - véritables "débris de la langue nationale" - et les noms de lieux où le basque est aujourd'hui parlé, tel doit être l'objectif d'une approche raisonnée de l'histoire linguistique de la Péninsule ibérique. Humboldt conçoit ses recherches en les ponctuant de questions qu'il ordonne de manière logique. $S^{`}$ il aboutit à la conclusion que le basque était la langue des habitants primitifs d'Espagne ${ }^{83}$, il se demande immédiatement si cette langue était ou non l'unique langue de ce pays, étant donné que les noms basques sont inégalement répartis en Espagne : "La plupart se présentent surtout chez les Vascons, puis chez les Turdules et les Turdétans de la Bétique" " L'exploration des mots désignant les entités collectives et géographiques suivantes - Basques, Espagne, Ibérie, Espagne - prend une signification toute particulière. Et Humboldt de dresser un constat paradoxal quant au niveau de connaissance anthropologique des peuples selon l'aire géographique à laquelle ils appartiennent: "Les deux nations de l'antiquité dotées de langues les plus complètes et de la littérature la plus florissante, la Grèce et Italie, partagent cette singuliere destinée que leurs habitants primitifs nous sont bien moins connus que ceux des contrées barbares" ". Leur perfection a été telle qu'elle a rejeté le passé dans l'obscurité et dans l'oubli. Ainsi en est-il de l'Italie qui ne manque pas, elle non plus, de noms d'origine basque.

En dessinant ainsi une carte des usages du basque dont le monde ibérique fut l'épicentre et qui a entretenu dans le passé lointain des relationss de proximité avec d'autres langues, Humboldt aboutit à une certitude fondamentale qui confirme la vieille théorie linguistique présente en Espagne depuis le XII siècle et magnifiée par les apologistes de la langue basque du XVIII" siècle: "Les anciens Ibères sont la souche des Basques actuels ; les lbères étaient répandus dans toute la péninsule, parlaient une seule et même langue et formaient plusieurs peuplades avec des dialectes divers. La langue basque était donc celle des habitants primitifs de l'Espagne, soit autochtones, soit venus dans ce pays à une époque antérieure à tout témoignage historique" ${ }^{86}$. En dépit de mélanges avec les Celtes, les Ibères n'ont pas connu "l'institut des druides et des bardes": "De là aussi sans doute moins de régularité dans la langue basque. surtout pour revenir des dérivés aux racines. Ailleurs, en effet, les druides et les bardes, seuls possesseurs de toute la science de leur époque, veillaient à maintenir la régularité de leur langue" ${ }^{87}$. Aussi la recherche sur le basque doit-elle se prolonger par une recherche comparative entre "les idiomes basque, breton et germanique" et avec les langues de l'antiquité : "On reconnaîtra ainsi celles de ces langues qui se distinguent du grec par des caractères marqués et on en fera le point de départ de nouvelles recherches" ${ }^{2}$. 
A côté mais avant l'antiquité grecque à laquelle Humboldt accorde un intérêt historique et philosophique majeur, se distingue l'antiquité basque, premier maillon de l'histoire primitive européenne; "Je la [langue basque] considère comme une langue purement européenne, une des plus anciennes, et si j'ose m'exprimer ainsi la plus ancienne de la partie du monde que nous habitons. C'est bien la langue d'un peuple antique, répandu sur un immense espace, et dont la destinée est étroitement liée à l'histoire primitive de l'Europe occidentale" ${ }^{89}$.

En multipliant les faits aptes à vérifier ses hypothèses, en critiquant les sources qu'il exhibe, Humboldt se veut historien en même temps que philologue, sans pourtant énoncer quelque théorie historique ou linguistique achevée, pour démontrer que le basque est le produit de l'histoire et non le produit d'un événement religieux (mythe de Tubal, descendant de Noé, venu s'installer en Espagne et premier bascophone) comme l'avaient imaginé les apologistes de la langue basque. L'héritier de l'Aüfklarung et le protestant qu'il est opèrent, pour la première fois, la démythologisation religieuse du passé de la langue basque. L'individualité du basque, il la forge à partir d'un double constat : son antiquité, d'une part, ses règles internes, d'autre part. De là, l'individualité de la nation basque qu'il approfondit au travers d'une étude sur l'histoire, l'ethnologie, la géographie et la psychologie des Basques. La mise en évidence de cette individualité du basque ne vise nullement à l'enfermer dans la spécificité de ses traits, mais à l'inscrire dans l'ordre de l'Universel figuré par l'humanit $e^{\circ \%}$. Le souci constant d'associer l'ordre de l'individuel et celui de l'universel signe la disposition intellectuelle sans doute majeure de Humboldt pour qui l'intelligibilité du réel historique relève de l'évidence scientifique élémentaire. Rétif aux constructions sur I'histoire, il recherche obstinément "le sens historique" ${ }^{91}$ en évitant de "forcer les événements" ${ }^{2}$. Partageant la passion de son temps pour la Grèce et la Rome antiques", il préfere l'Antiquité aux temps modernes et de manière particulière, l'antiquité grecque et romaine ${ }^{\mathrm{N}}$ par rapport aux autres civilisations "barbares". L'Antiquite connote le sens ou la proximité du début ; elle en tire sa perfection : "ce début [dit-i]] est aussi sa perfection" " $*$. Humboldt fait figurer le basque, en même temps que les langues celtique et germanique dans le cercle des langues choisies qui méritent et supportent une comparaison avec ces langues de l'Antiquité - le grec et le latin - qui symbolisent la perfection.

Dans un autre ouvrage consacré à la langue basque, et à ce jour non traduit, Berichtigungen und Zusätze zum ersten Abschnitte des Zwevten Bzandes des Mithridates über die Cantabrische oder Bashische Sprache ${ }^{\star}$, et publié plus tard, il rappelle comment après avoir dû s'éloigner de l'étude du basque pour des raisons professionnelles, l'occasion lui fut foumie d'y revenir à la faveur de la proposition du Professeur Vater de l' associer à son ambitieux projet d'écriture d'un "tableau universel des Iangues" " . Les paritions internes du Pays Basque suscitent ses premières remarques ; "La nation basque est tellement déchirée que le chercheur se trouve en difficulté dès qu il souhaite trouver une définition générale sur ce pays" " ${ }^{*}$. Les diversités dialectales l'indisposent particulièrement. L'absence d'œuvres littéraires l'oblige à travailler plutôt sur la langue parlée que sur la langue écrite. Une véritable attitude scientifique est ici déterminante car, seul, "le chercheur est capable de donner un aperçu de l"économie interne d une langue par rapport à la structure des mots" 9 ".

Cette précaution est nécessaire lorsque l'on aborde les comparaisons étymologiques qui peuvent entrainer des erreurs voire "la folie" ${ }^{10}$. Aussi convient-il de bien fixer la prononciation et les analogies internes car "chaque mot est un individu avec ses propres traits, comme un visage a ses yeux, son nez et sa bouche" wo! . 
Il rappelle aussi que la quête de traces antiques fut le motif essentiel de son voyage en Espagne : "chercher les traces des plus vieilles constitutions du peuple et des plus vieilles histoires à trouver toujours dans les anciens dictons et chants nationaux" ${ }^{102}$. De ces traces, la moisson ne fut pas, à ses yeux, abondante car ni les textes et ni la religion n'en contiennent. On les remarque dans les coutumes des vieux Basques, ou encore dans les corpus syntaxiques désignant les mois et les jour, les danses nationales et les contes" ${ }^{133}$. Tout en notant son origine douteuse, il rapporte le texte du "chant de Lelo", récit tragique d'un guerrier Lelo parti en guerre à l'étranger à l'époque romaine tandis que sa femme Tota le trompe avec un certain Zara qui la met enceinte. A son retour, Lelo est tué par les deux amants. Informé de ce méfait, le peuple se réunit et décide de chasser les deux amants criminels. Les chants nationaux connaîtront, on le sait au XIX' siècle, un grand succès, suscitant de nombreuses controverses autour de leur authenticité, beaucoup étant apocryphes. "Le chant de Lelo" n'échappera pas, comme on le verra plus loin, à de vives critiques émises, en particulier, par les interprètes de la vision républicaine de l'histoire de France : Julien Vinson et Jean Bladé assumeront ce rôle avec beaucoup de vigilance.

\section{b) Le peuple hasque ou le modèle du populaire}

Après les observations sur la langue qui incarne la dimension la plus expressive et la plus riche d'une nation, les études géographiques et ethnographiques constituent pour Humboldt l'autre versant d'une véritable et complète anthropologie. La nation basque lui offre le terrain idéal pour cet exercice qu'il veut exemplaire dans sa partie scientifique - saisie critique, dans le sens kantien, de la cohérence entre les traits constitutifs de la société basque - et généreux par sa portée politique - oeuvrer pour le bien de la nation basque.

Dans l'introduction de son ouvrage Die Vasken ${ }^{104}$, il rappelle les convictions philosophiques et les ambitions intellectuelles qui guident son entreprise : la perfectibilité de l'homme, l'intérêt d'une harmonie sociale entre le peuple et les élites, la valeur supérieure du primitif et de l'antique, la quête des traits spécifiques du populaire, la valeur ontologique de la coutume, la mise en évidence de l'exemplarité de la nation basque.

Humboldt commence par relever d'emblée la singularité du "peuple des Basques" qui a conservé au cours des siècles sa langue primitive et pour une large part son système coutumier, lesquels les auraient préservés du regard de l'observateur et de l'épée du conquérant ${ }^{10 s}$. Résistant à toutes les tempêtes historiques dont la première, la chute de I'empire romain, puis dans les temps plus récents, partagés de manière inégale entre deux "nations puissantes", les Basques n"ont nullement renoncé à "leur manière d'être". N'ayant connu aucun mélange avec leurs voisins, ils ont maintenu" leur caractère national" construit autour de leur esprit de liberté et d'indépendance tant souligné par les auteurs grecs et romains, et l'authenticité primitive des coutumes malgré les progrès accomplis par le luxe et le raffinement autour d'eux. Utilisant à nouveau la métaphore du torrent pour qualifier le cours de l'histoire, Humboldt observe que quelques autres peuples ont su maintenir leur langue maternelle et leurs coutumes "comme un tison" : ainsi en est-il des Bas-bretons de France, de leurs frères du pays de Galles, d'habitants des montagnes écossaises, de certaines populations d'Allemagne et de Suisse, des Estoniens et des Livoniens, de quelques autres peuples d'Italie et des Iles italiennes, restes d'autres nations jadis puissantes et étendues. Cependant, nul autre comme les Basques n"a su préserver jusqu'à ce jour une organisation politique indépendante et un "bien-être florissant" ${ }^{n}$, jouir des bienfaits de 
l'Europe éclairée sans pour autant "abandonner son caractère particulier et sa simplicité primitive" ${ }^{107}$. Humboldt applique ainsi la théorie du primitivisme, chère à l'Aufklärung, aux Basques qui l'illustrent par trois qualités fondamentales, l'authenticité, la simplicité et la modestie. Ces trois paradigmes spécifiant le monde ou l'état primitif, il les compare sans les opposer aux paradigmes culturels qui définissent l'époque "éclairée", la complexité, le raffinement et l' abondance, et qui se remarquent au sein des élites sociales. L'observation du populaire renvoie nécessairement au primitif qui symbolise le règne absolu du populaire. A partir de là, l'histoire se construit par l'accumulation et la stratification, c'est-à-dire par la structuration des rapports sociaux jusqu'à l'affirmation de divisions sociales.

Une explication scientifique doit être foumie pour rendre compte de la permanence. chez les Basques, de cette simplicité primitive. Humboldt la trouve dans la géographie. C'est en effet sa position géographique qui a protégé la nation basque contre les invasions, tout en favorisant les communications et le commerce avec le monde entier ; $c$ " est aussi dans sa position géographiquee "qu"il faut chercher la clé de toute son histoire, et en particulier de la plus lointaine" ${ }^{10}$. Les provinces basques peuvent esperer en Espagne et en France un accroissement de leur population et de leur bienêtre. Mais des influences diverses et fortes les préparent inexorablement à l'abandon de leur singularité nationale. La débasquisation est bien engagée et l'aire linguistique bascophone a tendance à se réfugier dans les zones montagneuses; de plus, celle-ci est "traitée comme par une mauvaise mère par la partie la plus illustre de la Nation" ${ }^{109}$. Le déclin du basque est prévisible et Humboldt d'imaginer qu" en moins d'un siècle, le basque aura peut-être disparu de la série des langues vivantes" " ${ }^{10}$. Il propose une conception déterministe de l'histoire selon laquelle, lorsque la société atteint "un certain degré de civilisation", les différences sont appelées à s'estomper et les petits peuples à se fondre dans les grandes masses ${ }^{111}$. Et pour orienter les nations vers des progrès intellectuellement importants et plus encore les protéger contre tout possible retour de la barbarie et de l'ignorance (maux les plus détestables qui soient pour un esprit éclairé), de grands moyens politiques s'avèrent nécessaires. Il est certes douloureux de constater le déclin d'un peuple même s'il s'agit d'une victime offerte pour le bonheur de l'humanité tout entière ; la douleur n'en est que plus grande quand on a affaire à la disparition d'une langue. Comment rester indifférent quand il s'agit de la disparition d'une langue "non affinée par la litterature" "2, s'imposant comme "l'unique expression du mode de penser d'un peuple rude" ? Langue populaire, le basque l'est assurément. Aussi observe-t-on spontanément et logiquement, chez les Basques, "plus d'exactitude saine du jugement que de formation scientifique, plus de sentiment naturellement chaud que de sensibilité raffiné" 113 . L'activité lettrée, d'après Humboldt, soumet une langue populaire à des distorsions qui affaiblissent ses capacités expressives. D'où la supériorité de l'usage du peuple sur celui de l'écrivain : "quand une langue accède à une formation littéraire et scientifique, elle s'écarte des mains du peuple et rarement gagne en énergie et richesse" "14. La vertu majeure de la langue populaire réside sans conteste dans sa spontanéité, ses débordements de sens, sa liberté créative, ses expressions hyperboliques alors que la langue littéraire et scientifique s'élabore en fonction des contraintes, des retenues et des limites imposées par le formalisme thématique. Celte spontanéité manifeste ses performances dans le transport "des nécessités plus immédiates", "des sensations plus naturelles", de "la fantaisie plus infantile". des "passions plus rudes" "l. Cependant, Humboldt évite de figer son destin, de l'enfermer dans l'espace social et la temporalité qui l'ont produite : avec le concours de générations ultérieures plus cultivẹes, la langue populaire peut accéder à un usage spirituel plus élevé ${ }^{116}$. Il croît à la plasticité et aux virtualités morales et intel- 
lectuelles de l"homme qui est "destiné à se perfectionner socialement" "17, de telle sorte que chaque individu se doit de s'agréger au groupe et que l'humain éclôt dans la simplicité de la nature et dans l'omementation la plus riche de la perfection éducative. Loin de n'exister qu'au travers de ses qualités propres, un caractère populaire, décidé ferme et vigoureux, exerce une influence morale et sociale majeure sur la totalité nationale, en permettant à la plus fine éducation d'une nation, à la vérité, à la force et au sens du devoir de s'imposer. Aussi l'étude des modalités d'expression des divisions sociales prend-elle ici une signification évidente ; en effet plus manifeste est la distance entre le peuple et les classes éclairées, moins perceptible se fait la formation des caractères populaires. Si la distance sociale neutralise les caractères populaires, la proximité sociale leur apporte un terreau favorable. Les Basques en foumissent une nouvelle fois l'illustration. Aux yeux d'Humboldt, les Basques, surtout ceux d'Espagne, connaissent un réel bien-être corporel sans lequel la prospérité morale est impossible. Outre les structures d'auto-gouvernement, ils montrent dans un même élan d'enthousiasme l'attachement à leur pays et à leur nation ; mêmes les gens fortunés, et ceux qui reçoivent en Castille des titres honorifiques demeurent fidèles de bon gré à leur patrie et entretiennent d'étroites relations avec le peuple dont ils conservent les coutumes et la langue. Une telle proximité sociale a pour effets de permettre la contamination partielle de la langue et des idées populaires par la Philosophie des Lumières et de rendre moins visibles les appartenances de classes dont les différences échappent complètement au regard du Basque ingénu. Humboldt entend insister sur les rapports harmonieux entre les classes sociales dans les provinces basques où le peuple fait montre d'une réelle "éducation naturelle" et où les personnes distinguées jouissent d'une réelle popularité, ce qui constraste avec les voisins tant français qu'espagnols. On peut seulement leur opposer, et de manière relative, le cas de petits cantons suisses où le sens de l'indépendance politique marque leur position externe et où une faible division sociale définit leur situation interne. En définitive, "la langue, l'organisation, les coutumes, la physionomie, et tout ce qui l'entoure, sans exclure l'aspect de leur pays, caractérisent les Basques comme "une souche pure et séparée" "18. Leurs données anthropologiques trouvent leur singularité dans leur isolement géographique, leur unicité morale et sociale ; à ce titre, ils illustrent exactement "le pur caractère de peuple" 119 en présentant l'ensemble des traits qui le fondent. Le voisinage avec la nature dans les principes éducatifs reste l'indice le plus significatif d'un état culturel bien compris. L'observation du présent invite à remonter le cours du temps pour déterminer les contours du passé. Une telle permanence et présence des structures du populaire parmi les Basques, qui signe une rare forme morale et sociale, légitime l'hypothèse d'une continuité entre le passé antique et le présent : "la particularité ethnique des Basques nous fait reculer vers les siècles lointains à l'époque antérieure à la domination romaine et cartagénoise, aux premiers habitants d'Espagne" Izo . Soumis aux contraintes et aux nécessaires évolutions historiques, cette particularité ethnique s'efface très progressivement ; elle subsistera néanmoins encore pendant longtemps. Cette situation suscite chez Humboldt, deux interrogations : l'une de nature historique, relative à l'origine de la souche et de la langue des Basques, l'autre de portée politique, concernant l'attitude de la monarchie espagnole à l'égard de la nation basque afin que l'Espagne puisse bénéficier de "sa force et de ses activités fructueuses" ${ }^{121}$. Le cas des Basques de France est l'objet d'une simple allusion à la République française qui n'accorde une "importance très secondaire" qu” aux seuls "districts basques" 122.

Pour la première, Humboldt note que l'esprit partisan et les choix arbitraires ont prévalu au détriment d'une analyse circonstanciée et solide. Les chercheurs étrangers ont travaillé à partir d'une connaissance insuffisante du basque tandis que les auteurs 
indigènes sont supects pour d'autres motifs. La seconde interrogation gagne à être considérée avec beaucoup d'attention dans la mesure où les situations de co-existence de plusieurs peuples au sein d'un même Etat ne sont pas rares. Force est de constater qu'à ce jour le génie politique s'évertue plutôt à se débarrasser des difficultés issues de cette diversité qu' à jouir des avantages constitutifs de cette singularité ${ }^{122}$. Des considérations analogues l'ont poussé à faire un voyage en Espagne, "lieu attirant pour les investigations sur la nation et la langue basques" " ${ }^{24}$. Si la finalité du voyage est bien d'ordre linguistique, Humboldt entend contribuer "pour sa part, à donner des idées plus correctes et exactes sur la nation et la langue basques que celles qui dominent aujourd'hui" ' ${ }^{\prime:}$. Cela passe par la mise en scène des Basques avec leurs coutumes et leur mode de vie. De là l'impontance qu'il attache à "leurs proverbes, leurs danses nationales, leur musique et leur poésie", témoins précieux de "leur mode de pensée et de vie" ${ }^{136}$. Ces pratiques-là sont l'expression polymorphe du génie et de l'exubérance de l'âme populaire ; à ce titre, elles composent le versant "culturel" de la spontanéité populaire et complètent le versant linguistique abordé précédemment. La richesse expressive du populaire explique la séduction qu'il ne manque pas d'exercer sur des esprits pétris de culture lettrée et savante. Face à l'esthétique aristocratique ou bourgeoise, prend place une esthétique du populaire construite selon les canons aristocratiques ou bourgeois. L'approche du grand aristocrate qu' est Humboldt constitue assurément un modele du genre : le XIX' siècle consacrera, on le sait, le succès d'une telle approche qui trouvera, en particulier dans le programme muséographique, sa plus forte traduction. En rappelant sans cesse sa volonté de faire oeuvre de science et conjointement en multipliant jugements de valeur, appréciations et observations sur les charmes et l'hétérodoxie du populaire, Humboldt fixe les modalités de constitution d'une science et d'une esthétique du populaire. L'aristocrate, le protestant et l'adepte de l'Aufklärung se retrouvent dans la défense et l'illustration, indifféremment, de la germanité et de la basquité. Humboldt confesse que l'image de la nation basque a marqué son âme à tout jamais, à tel point qu' il aurait aimé ériger un "monument" en témoignage "du respect et de l'amour" qu'elle lui a inspirés, concluant par ses mots le préambule de sa monographie ${ }^{127}$

Humboldt entreprend son périple à partir de Saint-Jean-de-Luz, privilégiant la partie côtière des Pays basques de France et d'Espagne, multipliant les observations de caractère géographique (le rôle des montagnes et des rivières dans la formation des mentalités), et de nature ethnographique sur le travail des femmes, les rapports entre les sexes, l'habitat et l'espace domestique, "l'organisation du pays", la fonction paradigmatique de la coutume, les représentations ethnotypiques des uns et des autres, avec pour objectif principal la mise en évidence et la démonstration de l'originalité des Basques dans leurs façons de structurer l'économie, la politique, la fête, leur imaginaire. en somme, la nation basque. Tout ceci souvent entrecoupé de développements philosophico-anthropologiques, parfois obscurs, autour de lieux communs de j'Aufklärung, de thèmes très présents dans les pensées herdiennes et fichtéennes, en particulier.

Humboldt reprend les catégories communes de l'époque pour désigner les Basques : Bizcaiens pour parler des Basques d'Espagne, Basques pour qualifier les Basques de France. C'est aux Basques que revient l'invention des techniques dans les opérations de pêche et d' approche des baleines ${ }^{128}$. La présence de "patriotes éclairés" (tels le comte de Peñaflorida, fondateur en 1763 à Vergara de la première société économique d'Espagne - la Real Sociedad Vascongada de los Amigos del País, ou le comte de Montehermoso à Vitoria) suscite son admiration. Il porte une attention très 
soutenue aux structures qui participent à "l'organisation du pays" : les corporations professionnelles telles que les cofradias, les entités territoriales de gestion administrative que sont les Hermandades (de l'allemand Germanus, selon Humboltd) en Alava. Celles-ci sont autant d'exemples d'autogouvemement local dont l'essence est supérieure à celle de structures plus générales. Les usages locaux, les micro-structures proches des individus - expressions de la société d'en-bas -, sont préférables aux concepts et aux directives venus d'en-haut (et en particulier de l'Etat). Ils foumissent aux individus des repères visibles et intelligibles, expérimentés et consacrés par la tradition; l'insertion de l'individu n'en est que plus facile, plus forte et plus durable. Constitutive et autre illustration de cette "organisation du pays", la multitude de caserios ou d'ante-iglesias (petits villages) qui caractérise si fortement le mode d'habitat en Pays Basque espagnol par rapport aux régions d'Espagne atteste clairement qu'en Viscaye, "on a su lutter contre la prépondérance des villes ${ }^{129}$. La nation s'édifie et se nourrit à partir des ressources du populaire paysan. Ces petites unités de vie apportent aux populations les cadres mentaux qui insufflent l'esprit d'indépendance, forgent les caractères, préparent à la morale de l'effor, socialisent l'amour de la nation et de la langue. Parmi les espaces naturels, la montagne a, sans conteste, la faveur de Humboldt, dont les propos moraux à son égard abondent ; la montagne permet, en effet, l'accès à l'excellence morale par la terrible discipline du corps et de l'esprit qu'elle suppose. Elle est, à bien des égards, J'incarnation de la Nature dans la plénitude de ses dimensions physique, philosophique et anthropologique. Humboldt ne fait nullement état de critiques anti-urbaines. Si la ville cumule les richesses et les symboles culturels et économiques - au cours de son voyage, il insiste sur le dynamisme commercial et industriel des villes du Pays basque -, la société paysanne conserve une supériorité ontologique (due à son statut de conservatoire du primitif et du populaire) ${ }^{1.2}$, qu expliquent son assise populaire et par voie de conséquence son ambition nationale ${ }^{131}$. Si en France, pour la Philosophie des Lumières, la société rurale/paysanne/populaire, "trace" de l'Ancien Régime, espace de l'obscurantisme, de l'étroitesse d'esprit et de relations sociales rigides et pesantes, se doit d'être civilisée par la ville, cité modèle des Lumières, en Allemagne pour l'Aufklärung, la société paysanne reste, au contraire, parée de toutes les vertus et n'a rien à devoir à la ville qui est loin de pouvoir synthétiser les principes d'une rationalité technique absolue. Comment ne pas évoquer la nature harmonieuse, en Pays basque, des relations entre le monde humain et le monde animal, illustrée notamment par la proximité physique du paysan avec son attelage de boeufs dont les têtes sont introduites, par une ouverture, dans sa cuisine ${ }^{132}$ ? Comment ne pas s'émerveiller devant la force paysanne observable chez les habitants des villages basques isolés et absente chez les habitants de nombre de provinces d'Espagne qui, eux, habitent plutôt en ville, "ce qui est préjudiciable à leur caractère" 133 ? Les autres observations faites par Humboldt sur l'espace domestique tentent toutes de cerner les cohérences et les valeurs inégalables qui inspirent la société paysanne basque ${ }^{1.44}$.

Autre figure hautement symbolique du populaire basque, la danse, à laquelle Humboldt réserve une dizaine de pages ${ }^{13 s}$ et dont il souligne la complexité de l'enchevêtrement des références et des sens : les rapports entre les classes sociales, le religieux et le païen, les relations entre les sexes, les mouvements indécents et la retenue dans l'expression, la part de l'authentique et la partie de l'emprunt, la différence entre la danse/divertissement -, et la danse/essence d'une nation.

Gestes. mouvements du corps, émotions, sensations, rythme, musique constituent tout en l'agrémentant le registre de la danse ou plutôt des danses car elles doivent être 
analysées dans la diversité des codes et des valeurs qu'elles véhiculent. Par la danse, derrière le formalisme des attitudes, s'exprime un jeu polysémique qui renseigne sur l'état social ou moral d'une société, sur son statut comme prétexte à une mise en scène et à une émotion coilectives ou simplement pratique "des classes inférieures du peuple" ${ }^{2.6}$. L'inventaire analytique des danses basques (dont les descriptions pour certaines, il le confesse, ont été établies à partir de témoignages) se veut sobre et précis. Si les carricadantza, chipiritaina et culadas, n'appellent aucune remarque spécifique, le fandango suscite par contre des commentaires, par ses origines non basques et par l'implication limite des règles de la morale. En effet, du fandango d'après certains d'origine castillane et américaine, porteur " $d$ " une voluptuosité lubrique" ${ }^{137}$ provenant de la nature du climat, Humboldt distingue, au prix d' une casuistique sophistiquée, le fandango du Pays basque qui, lui, "reste dépouillé de tout caractère de voluptuosité, est la danse naturelle rude, primitive pourrait-on dire, également indécente et obscène (non pas en général ni par essence, mais plutôt chez l'un ou l' autre des danseurs) mais nullement lubrique" ${ }^{138}$. Si l'éloge du populaire constitue l'un des thèmes centraux de la pensée humboldtienne, la lubricité semble marquer, seule, la limite extrême du populaire, et désigner la part non acceptable, troublante, sauvage et probablement non domesticable du populaire. Expression d'une pudeur personnelle ou acte de censure du populaire, nul doute que cet aspect heurte ou plutôt gêne autant le protestant que l'homme éclairé qu'il est. Heureusement, les Basques ont pu être préservés de cette marque expressive extrême pour deux raisons : par son origine extérieure au Pays basque, le fandango prend l' allure d'un "ajout exotique" ${ }^{19}$; il releve du "divertissement" et ne signe en aucun cas une dimension essentielle de la basquité : "les danses nationales authentiques du Pays basque portent toutes un caractère plus élevé et plus noble que celui du divertissement populaire en soi, et se distinguent beaucoup plus par le respect et la dignité, elles l'étaient encore davantage dans les temps passés quand la partie distinguée de la nation se distanciait moins qu'aujourd'hui du peuple et des coutumes locales, quand les autorités accordaient à la danse une attention plus soutenue ou quand les prêtres parlaient moins contre elles (les danses) et manifestaient moins leur zèle" ${ }^{*+1}$. De manière plutôt surprenante parce que inhabituelle, Humboldt constate, en les déplorant, l'existence d'écarts, entre les gens distingués et le peuple au sein de la société basque, et l'attitude hostile du clergé à l'égard des danses, objet d'un vieux contentieux avec l'Eglise depuis le concile de Trente. En même temps, il précise les contours de sa théorie du populaire ou de l'idéal-type populaire, d'une part, par la distinction entre l'ordre de l'accidentel (la danse-divertissement), et l'ordre de l'essence (la danse-pratique intrinsèque), entre l'origine endogène et l'origine exogène ; d'autre part, par la hiérarchisation axiologique de ces dimensions, à savoir la supériorité respective de l'ordre de l'essence et de l'endogène sur l'ordre de l'accidentel et l'exogène. De plus, l'ordre de l'essence se manifeste, dans le domaine de la danse, dans l'esthétique des formes, dans une sorte de theâtralité des mouvements produisant une émotion collective tandis que l'endogène se décline dans la diversité et la plasticité illimitée de formes locales qui, toutes, célebrent l'inventivité et le génie du peuple : "Seules les danses nationales exclusivement basques que l'on suppose d' origine plus anciennes, ressemblent plus à nos danses figurées, sont des représentations thêâtrales ou pour le dire ainsi des jeux de société" 'a!!

Chaque localité peut exhiber une danse propre qu'elle exécute habituellement. comme une sorte de marche solennelle lors d'événements importants dans la vie du village, tels que la fête du corps du Christ, le jour de la Saint-Jean ou la fête du Saint patron du lieu. La fécondité du populaire prend aụ Pays basque une dimension remarquable car aux danses déjà citées, s'ajoutent le zortzico" ["la danse la plus artistique" 
${ }^{1 * 2}$ ], l'acheridantza, le toalladantza, le trokiua, le jorraidantza et l'espatadantza, ces deux dernières danses "étant peut-être les résidus les plus antiques des coutumes" ${ }^{143}$. L'espatadantza a la particularité d'être exécutée par une trentaine de danseurs, au cou orné d'un scapulaire, tenant une épée ; Humboldt y discerne les éléments d'une scène guerrière paienne récupérée par l'Eglise et transformée en cérémonie religieuse. A plusieurs reprises, Humboldt parle de la solennité de ces danses ${ }^{1 / 4}$ en les différenciant de celles qui ressortent de l'initiative privée ${ }^{i 45}$, le premier terme recouvrant les notions du cérémonial de la forme et du sens collectifs, d'espace public, par opposition à la notion du privé, figuré par l'espace de l'individu. Cette césure entre le solennel et le privé correspond à une option philosophique majeure de l'idéologie de l'Aufklärung, c'est-à-dire celle de la primauté du collectif, du national sur l'individuel, celle de la subordination nécessaire de l'imaginaire individuel à l'imaginaire national désigné également par le concept "d'esprit public". L'expérience et le sens du collectif exigent le jugement des attitudes sociales qui excluent a priori l'esprit de facilité ; d'indifférence ou de fronde à l'égard des usages telle est bien la raison fondamentale du regard public ("la inspección publica"), celui-ci garantissant le respect de la coutume, "la rectitude et la force morales du caractère" ${ }^{14}$. A cet égard, de l'avis de Humboldt, la nation basque présente l'insigne singularité de cumuler toutes ces qualités et ainsi de dépasser toutes les autres nations. Le fait qu'il y avait jadis une solennité plus favorable à l'intégrité des coutumes" ${ }^{147}$ n'attente nullement à ce jugement global sur la basquité.

Enfin, une autre dimension du populaire aiguise l'attention de Humboldt, celle de ces productions orales que sont les contes et les fables, lesquels "sont toujours l'expression plus naturelle et plus particulière de la fantaisie nationale ${ }^{148}$. Il note que "nul peuple n'a poussé aussi loin, peut-être, la passion des contes que les Basques" ${ }^{149}$. Ils se réparissent en plusieurs genres dont ceux de los duendes ${ }^{1.0}$ et ceux de los imposibles (ceux des "impossibles" ou des impossibilités - telle l'histoire de la vie de celui qui n'est pas né). Urgente et utile, elle le serait la collecte de ces contes mais hélas, les individus cultes capable d'entreprendre un tel travail n'appartiennent pas au peuple et de plus ignorent ce patrimoine populaire ou répugnent à s'en occuper ! Cette collecte devrait être faite dans la langue basque elle-même car un recueil en espagnol affaiblirait la grâce des contes. Il en est ainsi de toute poésie populaire dont la traduction s'apparente à une dénaturation ou à une trahison. L'écriture de cette classe de textes rencontre les difficultés et les contraintes similaires à celles de la traduction.

La distance entre l'oraliture dont l'exubérance et la plasticité expressive sont stimulées par le génie de la langue, et l'écriture (ou la traduction) renvoie à la grande question philosophique et anthropologique, à savoir la distance entre la Nature et la Culture. Loin d'exprimer une opposition entre une logique du désordre et une logique de l'ordre, la première renvoie à une créativité fondée sur la spontanéité, le débordement des sens, l'imagination, la fantaisie, la seconde renvoie à une créativité soumise à des règles, à des codes, à l'instrumentalisation de la raison. Ce couple conceptuel recouvre et propose une lecture des séquences historiques ainsi réparties : celle des temps primitifs où règne la Nature et celle des Temps modernes où officie la Culture. Par le biais de ces premières analyses sur le thème du conte, prend forme un nouveau corpus de savoirs auquel les Frères Grimm, en particulier, donneront ultérieurement un statut littêraire.

Particularité insigne des contes basques, Humboldt décèle une ressemblance entre les récits basques et les fables grecques. Cela ne justifie pas que l'on envisage une origine commune antique à partir de vestiges à moitié disparus. Point de précipitation, prévient Humboldt qui peut apporter une explication logique. "La fantaisie et les pas- 
sions humaines" "sont un bien commun à tous les peuples et trouvent dans la civilisation rurale le terreau social de prédilection. Le génie de chaque peuple produit selon les circonstances et les périodes historiques les ressemblances ou les différences conformément, néanmoins, au postulat fondamental que l'inventivité s'exprime dans la multiplicité et l'hétérogénéité, le destin culturel de tout corpus de contes étant de traduire ces deux aspects dont la transfiguration symbolique se résume et se réalise, à travers l"unité consentie de toutes les classes constitutives d'une société, dans la nation.

Humboldt achève son voyage en revenant à son point de dépar, le Pays basque français, ce qui lui donne le prétexte pour se livrer à l'ethnographie comparée des Basques de France, des Français, des Basques d'Espagne (appelés par l'opinion de l'époque les "Bizcaiens") et des Espagnols, en s'appuyant sur les représentations collectives produites de part et d'autres. Les ressemblances et les contrastes sont notés avec subtilité et avec le souci de ne blesser personne, en équilibrant les éloges et les réserves. Les Basques de France font preuve "de force et d'agilité du corps, [d]"un niveau élevé d'imagination, [d]'une tendance constante aux idées générales, parfois singulières et caricaturales, [de] sentiment tendre, toujours prompt et de mouvement facile, et [d]'un esprit de liberté qui se manifeste devant toute contrainte sociale : mais en même temps, on leur reproche frivolité, une inclination très forte au divertissement et un changement constant dans les penchants" 15? Les Bizcaiens, eux "jouissent parmi leurs compatriotes et leurs voisins d'une réputation plus solide. Agilité et force corporelle, intelligence de l'esprit et noble sentiment de liberté qu'on ne leur conteste pas", et passent nettement pour constituer "une nation réfléchie, travailleuse, persévérante ayant de la fermeté dans ses projets et qui se soumet de bonne grâce à une contrainte nécessaire" ${ }^{163}$. L'examen des contrastes s'avère en réalité plus intéressant et plus pertinent, même si les références stéréotypiques ne manquent pas : frivolité et esprit frondeur pour les uns, pesanteur et conformisme pour les autres. Si "le caractère basque mélangé au français (comme cela se remarque chez les personnes qui ont fait la littérature) donne finalement un attrait indescriptible" ${ }^{1 \leqslant 4}$, qui jette "une parure de fantaisie à la fois sur l'esprit et le sentiment" "s", tandis que les Basques d'Espagne "ont manifestement quelque chose de plus lent, de plus pesant dans leur être : leurs traits physiques expriment moins de mobilité, moins de subtilité, moins d'esprit et de fantaisie" ${ }^{1.6}$. La comparaison des attitudes permet aussi de relativiser les singularités ou de déplorer les effets de miroir: "Les Basques français sont simplement une petite souche rare dans un grand pays supérieur à eux par tous les styles" ${ }^{157}$. Des cheminements politiques et culturels dissemblables font que les Basques passent inaperçus en France et jouissent, par contre, d'une grande notoriété en Espagne : "De manière générale, l'esprit national en France n'a jamais été décliné sur le mode provincial. comme cela se fait en Espagne. Les Basques se comparent toujours aux Français avec lesquels ils ne peuvent disputer une supériorité sur aucun aspect ; les Biscayens le font avec les Espagnols auxquels ils sont notoirement supérieurs sur plusieurs aspects ${ }^{* 1.4 x}$. Et après avoir répété les analyses déjà établies sur les uns et les autres, en guise de premiers balbutiements d"une Völkerpsychologie, il arrive à la certitude "que les Basques constituent une Nation et que la similitude de leurs traits caractéristiques peut dériver de l'égalité de leur origine" 159. L'approche comparative se fait plus micro-territoriale et prend, comme objet d'investigation, dans un premier temps, les relations à Bayonne entre Gascons et basques. Ces derniers, dit-il, connaissent la situation de tout groupe minoritaire confronté à un groupe majoritaire et ont la réputation d'être des "filous. des rusés et des poltrons" "indis que l'intérieur du Pays Basque passe pour être un 
"pays sauvage" ${ }^{16 t}$. Mais rétorque finement et avec indulgence Humboldt, "heureusement, la part éclairée de la nation ignore ces jugements", et si "les reproches de sagacité et d" astuce ont quelque fondement", c'est que "le Basque (surtout le Basque français) est plus rapide et habile que grand et fort et que, comme peuple de montagne, ils eurent à lutter, en d'autres temps, contre des ennemis extérieurs et qu'aujourd'hui ; comme frontaliers, ils sont confrontés aux limitations peu satisfaisantes pour avoir un travail de contrebande" ${ }^{62}$. Il rappelle succinctement les modalités d'administration des provinces basques françaises (Labourd, Basse Navarre, Soule) sous l' Ancien Régime, en concluant ainsi : "comme la Révolution Française a supprimé toutes ces constitutions particulières, il serait peu pratique de nous arrêter davantage sur leur exposé" ${ }^{16 \text { ? }}$. Il voit ainsi dans la rupture révolutionnaire un événement désormais déterminant pour le cours de l'histoire, qui recueille son assentiment et qui, de ce fait, n'appelle aucune remarque critique. Il illustre ainsi une nouvelle politique inspirée par l'Aufklärung, selon lequel les "constitutions" - c'est-à-dire l'armature juridique - fondent et expliquent le sens et la qualité de toute société.

L'approche comparative psychologisante s'achève avec les Souletins qui, dit-il, croient posséder plus de goût et de finesse que leurs compatriotes et font montre d'un fort penchant, au sein de toutes les classes sociales, pour la poésie et la musique. Malheureusement, ce penchant si évident au sein de ce petit peuple auquel font défaut "tous les instruments nationaux intéressants" pour inspirer son imagination, est appelé à "se dégénérer en plaisanteries creuses et en bavardages poétiques" 164 . In remarque les représentations théâtrales pastoralières jouées par des acteurs des deux sexes et généralement illettrés. Il note la difficulté à établir des relations matrimoniales avec les Béarnais et se plaît à voir dans la Soule "un espace de transition des Basques aux Gascons - ou si l'on considère davantage la similitude des caractères que la proximité géographique - aux Provençaux" ${ }^{16}$. Et le livre de se terminer par un aveu d'impuissance : la réflexion anthropologique prolongée par une esquisse de Völkerpsychologie ${ }^{100}$ des provinces basques n'en continue pas moins de se heurter aux trois questions centrales qui structurent la pensée historique : "le où, le quand et le comment" ${ }^{167}$. Bien qu'animé par l'esprit positif, le voyage dans la basquité n'arrive pas à entamer l'obscurité qui tout à la fois la définit et la rend inaccessible.

La conjonction de cette ethnographie et de cette psychologie comparee vise à définir "l'esprit" du peuple basque - force de caractère, élévation des sentiments, attachement aux constitutions locales, goût du travail, esprit d'indépendance, sentiment communautaire fondé sur la solidarité entre toutes les composantes sociales -, de manière empirique, et pour l'étude des principes déterminants principaux (le sens et la puissance de la coutume, de la géographie). Cette approche, constitutive de l'Aufklärung, fondée sur les théories de la relativité culturelle et de "l'individualité nationale" aura en France, au XIX' siècle, un prolongement mais selon des voies très distinctes : la perspective ethnographique/folklorisante développant une analyse réductrice de la théorie du relativisme culturel, tandis que le courant de défense du droit naturel basque (celui-là même qui utilisera la bannière de la tradition basque contre toutes les expressions culturelles de la Modernité) défendra plutôt la théorie de l'individualité nationale des Basques. Par contre, dans les pays germaniques, les travaux menés par Humboldt autour de la basquité auront des conséquences importantes et durables en particulier, en confortant au sein des universités allemandes et autrichiennes un intérêt prononcé pour la linguistique basque. 


\section{III - L'écho de la basquité en Allemagne au XIX' siècle}

La constitution de la science linguistique au XIX' siècle n'est pas étrangère à l'impressionnant mouvement de publication de centaines d'ouvrages, d'articles et de mémoires sur le basque par une cohorte de linguistes, certains prestigieux, d'autres moins, souvent avec l'appui des institutions les plus doctes telles que les Académies des sciences de Berlin, de Vienne et d'Amsterdam. Parmi ces linguistes, les allemands F. Müller et Th. Linschmann, l'autrichien H. Schuchardt, les hollandais Ulhenbeck et Van Eys donneront aux intuitions fécondes de Humboldt un très notable développement et parfois aussi un démenti à certaines de ces hypothèses, fait prévisible après trois quarts de siècle d'accumulation des recherches linguistiques.

Parmi ces bascologues éminents, Hugo Schuchardt mérite assurément une attention particulière tant par la nouveauté et l'importance de ses travaux dans le domaine, en particulier, de la morphologie basque et plus précisément dans l'étude des procédés grammaticaux et des catégories grammaticales du basque. Humboldt et Schuchardt ont un point commun : celui d'avoir vu leurs passions linguistiques naître à la suite de lectures d ouvrages (grammaires et dictionnaires) traitant du basque. Très tôt, Schuchardt ${ }^{1 / 8}$ prend connaissance dans la bibliothèque de son Gotha natal, avec curiosité, de la Gramatica de las lenguas romanicas, de Friedrich Diez, et de la grammaire basque du jésuite Larramendi, dont l'étrange titre El imposible Vencido (1729) avait de quoi exciter la curiosité du lecteur. Fort de ces premières lectures, il entreprit les études universitaires et se dédia pendant quelques annés à la linguistique romane. Et quelques années après sa nomination, en 1876, à l'Université de Graz, il opte pour le retour à ses préoccupations de jeunesse et vient séjoumer, l'été 1887, à Sare, village basque français pour y apprendre le basque. Sejour bien fécond si l'on peut en juger par limportance des apports de Schuchardt à la connaissance des procédés grammaticaux et des catégories grammaticales du basque. En réalité, ses compétences linguistiques étaient aussi étendues que diverses. "Père incontesté des études de pidgin créole" ${ }^{1 * 9}$, il s"intéresse à bien d"autres langues comme le hongrois et des langues indoeuropéennes telles que l'albanais ou le slovène. S'agissant du basque, il lance l'hypothèse d'une parenté possible entre celui-ci et les langues caucasiques ${ }^{170}$. Ses propositions novatrices se remarquent dans l'étude des pronoms personnels (rejoignant sur plusieurs points un autre linguiste allemand Linschmann), de la déclinaison du basque similaire à bien des égards à celle du latin et de l'allemand (le hollandais Van Eys contestant l'existence d'une telle déclinaison), de la conjugaison à laquelle il consacre le plus d'analyse. Après avoir étudié les travaux de ses prédecesseurs et s'être penché sur les paradigmes des grammaires, il publie en 1893, un mémoire de près de 80 pages intitulé "Sur l'origine des formes de relation du verbe basque" " $"$. La compréhension du travail de Schuchardt mérite le rappel de la situation générale de la linguistique en Europe, au XIX' siècle. Après les grandes synthèses romantiques (Bopp, Humboldt), ou naturalistes (Schleicher), on en vient à l'étude positive d'une langue déterminée, position défendue par les Junggrammatiker ou "néo-grammairiens" " comparatisme avait abouti d'une part à la généalogie des langues indoeuropéennes. d'autre part, au classement des langues à l'intérieur de chaque famille (germanique avec Grimm, romane avec Diez, slave avec Schleicher. etc.). Il convient de mettre à l'épreuve les thèses historico-comparatives. le développement de la philologie des langues particulières (allemande avec W. Scherer, française avec P. Meyer et G. Paris. etc.) correspond à cette exigence.

Parallèlement à ces restructurations intradisciplinaires, se constituent, autour des années 80 , des enjeux épistémologiques tenant à la pertinence du principe de "Yinex- 
ceptibilité" des lois phonétiques dans le cadre d'une langue donnée, défendue par les jeunes grammairiens de Leipzig. Applicable aux ensembles linguistiques constitués, qu en est-il de ce principe avec les langues situées sur leur marge ? Parmi les études consacrées à ces dernières et plus précisément aux langues en contact (d'où l'intérêt pour les langues créoles), celles de Schuchardt sont estimées présenter une valeur fondatrice ${ }^{17}$. La "querelle des lois phonétiques" connaît son point culminant autour des années 1880 , querelle souvent réduite à une confrontation entre les jeunes linguistes de Leipzig et les "vieux grammairiens" 174 .

S'il décèle des emprunts romans dans la langue basque - une analyse qu'il mettra lui-même en cause -, sa fierté intellectuelle s'exercera dans son mémoire sur le verbe basque. Il confie à l'érudit anglais Dodgson, dans une lettre datée du 29 janvier 1893 , que "la conjugaison basque est l'une des choses les plus difficiles et les plus embrouillées de toute la linguistique et il donne à cela deux raisons : la première, c'est que le nombre des formes est extraordinairement grand (il déclare les avoir toutes utilisées et en avoir examiné au moins cinquante mille), et la deuxième, c'est qu'il faut tenir compte d'un grand nombre de facteurs ayant évolué et s'étant enchevêtrés dans les directions les plus variées" "175. D'après le linguiste G. Lacombe, le livre ne suscitera pas l'intérêt qu'il aurait dû soulever, sans doute parce qu'il n'avait été ni compris, ni lu.

L'intérêt profond des linguistes allemands sera à l'origine de la fondation à Berlin, en 1886, de la Baskischen Gesellschaft '("Société basque"), avec le concours de spécialistes réputés tels que les Professeurs Mahn, Th. Linchmann, Président de la Société (co-auteur avec Schuchardt de travaux sur le basque) et K. Hannemann, Secrétaire de rédaction de l'organe de la Société, la revue Euskara (Organ Fûr die Interesen der Baskischen Gesellschaft). La raison sociale de la société est ainsi définie : "Comme but principal la société se propose de stimuler l'intérêt que l'idiome des Basques est en droit d'inspire; d'en vulgariser la connaissance, afin d'augmenter sans cesse le nombre des personnes en état d'apprécier cette incomparable langue, et disposées à se consacrer à son étude; de soumettre à ses investigations la littérature, les légendes. les moeurs et usages, etc., des Basques et de leur pays, ainsi que les origines des Ibères. leurs ancêtres ; afin de prendre et de donner connaissance de toutes les publications et découvertes nouvelles sur le domaine de la langue basque et de leurs traditions" $1 \%$.

Les attitudes admiratives et généreuses des fondateurs de la Baskischen Gesellschaft rappellent la chaleur des propos de Humboltd à l'égard du basque ; son nom est d'ailleurs fréquemment évoqué dans les pages de la revue. Si l'intention des fondateurs est de créer un organe de liaison entre bascologues d'Allemagne et d'Ailleurs, la revue assurera cette fonction de manière peu probante, malgré la qualité des collaborations, avant d'arrêter la publication peu après. Le lancement en 1907 de la Revue Internationale des Etudes Basques répondra, plus tard, à la volonté d"organiser simultanément une liaison et une visibilité internationales des études basques. Néanmoins. la création de la Baskischen Gesellschafi aura marqué non seulement l' aitachement de la pensée académique allemande à la bascologie, mais aussi, de manière plus générale, l'existence d'une sorte de complicité opératoire à des degrés divers (malgré le bombardement de Gernica !) entre la germanité et la basquité, que d'aucuns ne manqueront pas de solliciter à des fins qui ont peu à voir avec la science. 


\section{NOTES}

1. J.Y. Guiomar. "De l'Allcmagne et de la France. Les faux semblants d'une opposition" dans le Débas, Gallimard, $n^{\circ} 84$, mars-avni, 1995, pp. $70-88$.

2. Ch. Pouthas. Les Révolutions de 1848 en Europe. Paris, CDU, 1952, p. 50-51.

3. Voir a ce sujet $\mathrm{H}$. Aarsleff, "The tradition of Condillac: The problem of the origin of langage in the 18 th contury and the dcbats in the berlin Academy before Herder" dans Studies in the History of Linguisticr. Indiana University-Press, Bloomington, 1974.

4. M. Duchet, Anthropologie et histoire au siècle des Lumières, post-face de Claude Blanckaert, Albin Michcl, 1995. p. 566.

Pour une connaissance des Lumières allcmandes, voir E. Cassirer. Die philodophie der Aufklärung, Tübingen, 1972, ou La philosophie des Lumières, trad. française par P. Quillet, Paris. Fayard, $1966 ; \mathrm{H}$. Brunschwig. La crise de l'Etat prissien d la fin du XVIII' siècle et la genèse de la mentalité romantique. Paris, PUF, 1947 ; M. Schaub. " $L$ 'Aufklärung et la problématique de la langue ; enjeux culturels et universalité scientifique" dans Recherches sur la philosophie et le langage - la philosophie dans sa langue. Université des Sciences Sociales de Grenoble, n²3, 1983, pp. 155-177.

5. J.G. Herder. Journal de mon volage en l an I769. Aubicr. 1942, traduction française de M. Rouche : voir M. Schaub, article cité, p. 156.

6. J.G. Herder. Auch cine Philosophie der Geschichte zur Bildung der Menschheit, Bevtrag iu vielen Bevträgen des jalthlinders, 1774, traduit par M. Rouché, Une autre philosophie de l histoire pour contribuer à l'éducation de l'humanité. Contribution à beaucoup de contributions du siecle, Aubier, 1943. Celuici ćcrit : "Si des le début, il n'a pas ćté écrit en vain, on voit que la formation et la croissance d'unc nation n'cst jamais autre chose qu une ocuvre du destin ; le résultat de mille causes concourantes et en quelque sorte de l'elément enticr dans lequel clles vivent", p. 253, ou encore "Je vois une grande oeuvre sans nom". p. 305 .

7. J.G. Herder. ibidem. "La longue. l'ćternelle nuit fut éclairée par le matin : ce fut la Reforme. la Renaissance des arts, des sciences. des moeurs ! La lie se déposa. ct alors ful - notre pensée à nous ! Notre civilisation ! Notre philosophie ! On commençait à penser comme nous pensons aujourd'hui : on n'etait plus barbare", p. 231.

8. La fin de l'ouvrage réserve a Voltaire un véritable réquisitoire tempéré par de l'admiration : " $L$ 'cerivain de cent ans qui, sans contestation ni contradiction a agi cn monarque sur son siècle - qui, de Lisbonne au Kamtchaka, de la Zemble aux colonics de l'Inde est lu, appris, admire et, qui plus est, suivi avec son langage, ses cents talents divers d'affabulation, avec sa facilité, avec son jaillissement d'idécs toutcs flcurics - sunout du fait qu'il cst né où il fallait pour utiliser le monde. utiliser prédécesseurs et rivaux. utiliser occasions et points de depart. surtout les faiblesses les plus profitables des plus belles fianctes de son tcmps. Ics souvcrains de toutc l'Europe - $c c$ grand Ecrivain. que n'a-t-il pas fait sans aucun doutc pour Ic plus grand bien du siècle ! Il a répandu la Lumic̀rc. cc qu' on appelle la philosophic de l'humanité. la tolerance. la facilité à penser par soi-même. un scintillement de vertu sous cent figures aimables, des petits penchants humains dilués et édulcorés - en tant qu "ćcrivain sans nul doutc, il est au plus haut sommet du siecle ! - Mais en même temps que tout cclá. quelle miscrable frivolite, que de faiblesse, d'incertitude et de froideur ! Qucllc absence de profondeur, de plan arrêté, que de scepticisme à l'égard de la vertu. du bonhcur et du méritc ! Que dc choses son rirc spirituel a balayées sans avoir voulu en partie les balayer ! - Que de doux liens, agrćables et nécessaires. il a rompus d'une main criminelle, sans nous donner quoi que ce soit qui pût les remplacer pour nous. qui ne résidons pas tous au Château de Fernay ? (...)". J.G. Herder, Pour une autre Philosoptie de l'histoire. ouvrage cité. p. 361 el 363.

9. J.G. Herder. ouvrage cite. p. 301.

10. J.G. Herder. ouvrage déjà cité. notamment p. 229 el suivantes.

11. Voir à ce suję M. Schaub, an. cité. p. 164

12. J.G. Herder. ouvrage cite. p. 175.

13. Ibidem. p. 185.

14. J.G. Herder. ouvrage cité, p. 195.

15. Idcm.

16. Ibidem. p. 215.

17. $I \mathrm{dcm}$. 
18. Ibidcm. p. 219.

19. Ibidem. p. 219 et 221.

20. Ibidcm. p. 219.

2]. Ibidcm, p. 221.

22. Idcm.

23. Ibidem. p 259

24. Ibidcm. p. 261.

25. Ibidem, p. 263.

26. Idem.

27. Idem.

28. Ibidem, p 271

29. J.G. Herder, Fragments. Ed. Suphan, 1767, t. I, p. 366.

30. J.G. Herder, Une autre philosophiue de Ihistoire, ouvrage cité. p. 287.

31. Ibidem, p. 123.

32. Ibidem. voir commentaire de M. Rouche, p. 54 et suivantes.

33. Ibidem, p. 25.

34. C'est unc idéc similaire greffée sur une approche critique des considérations ethnocentristes qu' $\mathrm{ex}$ mosc Montesquieu dans le premier chapitre de son Essai sur les mozurs: "Le grand malentendu sur lcs rites de la Chinc est venu de ce que nous avons jugé leurs usages par les nôtres : car nous portons au bout du monde les préjugés de notre esprit contentieux". De son côte, Adam Ferguson. déclarait déjà dans son Essai sur la société civile (1767) : "Nous avons toujours peine à concevoir comment l'espece humaine peut subsister sous l'empire de moeurs el de coutumes absolument différentes des nôtres et nous sommes portés à cxagérer lcs malhcurs des temps de barbarie (...). Mais chaque âge a ses consolations ainsi que ses peines" (Lraduction française de 1783 . Paris. 2“ partie. chap. 3. p. 292),

M. Rouché résume ainsi les sinuosités voirc les paradoxes de la pensée herdérienne : "Il a élargi en une philosophie de l'Histoire universelle la critique litteraire moderniste et relativiste de Saint-Evremont ct de I'Abbe du Bos : le sens de la relativité historique d'Adam Ferguson et de Montesquieu dans ses "Considérations sur les causes de la grandeur el de la decadence des romains. de la relativité géographiquc et cthnique de Voltaire et encore Montesquieu dans L'Esprit des Lois, qui constitue une des principales sources. à la fois négative et positive, de Une autre philosophie de l'histoire", dans J.G. Herder, ouvrage cité. p. 101.

35. Cette idec de l'irréversibilité de l'histoire. Herder l'illustre encore dans ses recommandations aux prètes de son temps de chanter non pas comme Homdre en son temps, mais comme Homère chantcrait s il revenait parmi cux, Fragments (1767-1768), t. II, p. 258 et suivantes.

36. J.G. Herder. ouvrage cite. p. 315.

37. Il convient de ne pas se méprendre sur le sens du populaire cher Herder, lequel n'a pas unc connotation socialc (ic populaire s'opposant au bourgeois ou à l'aristocratique) mais une signification culturelie (le populaire se distinguant du savant). De là, son effort pour la promotion d'une Naturpoesie ou poésic "naturcllc" a la place d"une Kunstdichtung ou pocsic savante. La Naturpoesie s'enracine dans l'âme pnpulairc et appartient à l'utopic de l'àge d'or de l'humanite. Voir à ce propos W. Bruickner, "Histoirc de la Volkskunde" dans El/hnologies en miroir. Essais réunis par Isaac Chiva et Utz Jeggle, Ed. dc la Maison des Sciences de l'Homme, Paris. 1987. pp. 223-247, p. 244.

38. Ces idécs étant clles-mêmes animées par unc autre, sous-jacente, celle de la noblesse de la culture populairc (alienande) par rapport au modèle dominant de la litterature française classique de Cour.

39. L'ouvre et la penséc de Herder posent de manicre excmplaire les problemes habituels de I histoirc des idécs. nolc G. Raulet : "Ia différence entrc Lumières et Aufklärung el la spécificité de l'Allfklärung". dans Aufklürung. Les Lumieres allemandes. Textes et commentaires par E. Raulet, Flammarion. 1995. p. 236.

40. Un excmple éloquent est foumi par la charge d'A. Finkielkraut contre les conséquences de la théoric du Volksgeist dans La défaite de la pensée. Gallimard, 1987. Reprenant à son compte les rcproches de Julien Benda - contenus dans La trahison der clercs (1926) - contre la société pensante européenne tentéc de sc complaire dans le spectacle de ses propres oeuvres, de sa propre culture, et la recherche de ses particularismes locaux. A. Finkiclkraut voit une filiation directe entre le relativisme anthropologique de Herder ct toutes les philosophies ultéricures de la contre-révolution: voir cn particulicr de la page 15 à 52. 
Par ailleurs. observe Pierre Pénisson, "On s'est servi de Herder (1744-1803) pour revendiquer le particularisme contre l'universel. le sentiment contre la raison, le romantisme contre le classicisme, le national ct l'origincl contre l'etranger. Par conséquent, on ne pouvait trouver en lui, bon gré, mal gré, que des discriminations. Or loin d'opposer les langues, les peuples, les nations, les cultures, toute l'oeuvre de Herder consiste à meture en relation, à rassembler "la voix du peuple de l'humanité disséminee". Ne cessant de rapporter les epoques et les langues les unes aux autres. Herder développe une philosophie de la traduction. Dans l'horizon intellectuel de la fin du XVIIr' siecle, c'est bien chez lui plutôt que chez Voltaire. Goethe ou Kant quc se produisent et unc libération et une crise de la langue" ; dans Johann Gonfried Herder, Paris, Ed. du Cerf, 1992, page de couverture de l'ouvrage.

41. Voir à ce sujch. P. Bidart. "Peru Abarca ; espace imaginairc et paradigme perdu" dans la Production sociale des espaces. sous la direction de P. Bidart, Université de Pau et des Pays de l'Adour, 1986, pp. 2747.

42. W. Moser, "Herder ct la toupie des origines", dans Mvthe de lorigine des langues. Revue des Sciences Humaines, Lille III, 1977-2. pp. 205-226, p. 213.

43. Idem.

44. Citéc par W. Brüchner dans Ethnologies en miroir, ouvrage déjà citê, p. 226.

De la Volkslainde. W. Brüchner dit "qu' elle est enfant de la germanistique, cest-à-dire de la germanolngie (Deutschumswissenschaft)" (p. 224); les Frères Grimm étant les premières grandes figures de cette Volkskunde.

Celle-ci fait Ccho au Folkjore anglais, tandis qu' $\mathrm{cn}$ France de fortes réticences, surtout d'ordre ideologiquc, ont empeché la formation d'un courant scientifiquc similaire, situation for justement analysce par I. Chiva : "A la différence de la plupart des autres pays europécns et pour des raisons complexes qui tiennent à l' histoire politique et culturelie et à celles des institutions scientifiques, le folklore n'a jamais pris vraiment en France (a de rares exceptions ponctuclles près), dans l'Universitc ou dans quelque autre grande institution publique. Au moment où les sciences sociales allaient s' institutionnaliser, lorsqu'elle connaitra son épanouissement universitaire, l'école de l'Année Sociologique a manifesté une opposition idéologique décisivc. basće sur le rejet du rôle attribue à la tradition el du folklore tel que l'incarnaient en particulier un P. Saintyves $\mathrm{ct}$ un $\mathrm{P}$. Sćbillot ; leur étaient cn même temps reprochés leur opposition idéologique à l'unjfication républicaine, leur catholicisme militant. le rôle excessif qu'ils accordaient à l'esthétique et à l' ${ }^{\prime} \mathrm{cm}$ blématique", dans Ethnologies en miroir. ouvrage cité, p. 30.

45. F.G. Herder, ouvrage cite, pp. 285-287.

46. Voir à ce propos W. Krauss. Zur Anthropologie der Aufklärung, Berlin. 1978 ; U. Ricken. "Linguistik und Naturwissenschaft in der Aufklärung", Nova Acta Lcopoldina", NF, $\mathrm{n}^{\circ}$ 230, 1978 : L. Formigari. Linguistica e antropologia nel secondo settecento, Massina, 1972 ; M. Duchet, Anthropologic el Histoire au siècles des Lumières, ouv. déjà cite ; U. Ricken, Grammaire et philosophie au siècle des Lumière's. Publications de l'Université de Lille, 1978.

47. On connaît les propos célebres de Rivarol : “(...) Ce qui distingue notre langue des langucs anciennes et modemes. c'est l'ordre et la construction de la phrase (...). Le Français, par un privilège unique. est scul reste fidd̨lc à l'ordre direct. comme s'il etait toutc raison (...) ; la syntaxe française est incorruptible. C'cst de là que résulte cette admirable clanć, base étcrnelle de notre langue. Ce qui n'est pas clair n'cst pas français ; cc qui n'est pas clair est encore anglais. italicn. grec ou latin", Le discours sur l'universalité de la langue française. Ed. Suran. Paris. 1930. p. 253.

Il n'est pas jnutilc de rappeler que ce Discours constituait la réponse au concours lancé. en 1783. par l'Académic des Sciences et des Lettres de Berín avec le sujet suivant: "Qu'est-ce qui a rendu la langue française la langue universelle de l'Europe ? Par où méritc-t-clle cette prétogative ? Peut-on présumer qu "clle la conscrve ?". Rivarol paragera le prix de l'Académie avec Johan Schwab.

48. Dans la mesure où cette théorie livre une perspective évolutionniste. clle suscitera des débats d'ordre lcxicologique autour des questions de synonymie er de néologie.

Les relations cntre néologisme ct politique (comme d'une manière générale entre linguistique et poli(ique) méritent une atention spéciale dès lors qu'il s'agit d'affirmer une volonte culturclle : il n'est que de voir le rôlc autribué par le fondateur du nationalisme hasquc, Sabino Arana Goiri, à l'activité néologistique. dans son souci đarchaïsation de la langue basque sclon les mêmes règles que cclles régissant les conditions d'intégration socialc, à savoir l'obligation d'attestation de l'apparenance plurigénérationnelle à la société localc.

49. U. Ricken. ouvrage cite. p. 180 et 181.

50. Cite par G. Raulct, Alifklärung. Les lumieres allemandes, Flammarion. 1995. p. 109. 


\section{Ibidem. p. 117.}

52. Le sens ou le principe communautaire se fondent sur l'cxistence de liens effectifs ou affectifs dans la constitution des formes primitives des sociétés. Cet aspect pose le probleme plus global du fondement des constitutions civiles et plus précisément celui du passage de la référence au droit naturel chrétien au droit naturcl rationnel, grâce en particulicr à la Réformc. Les deux contributions initiatrices dévcloppant l'idec du Contrat furcnt le De jure belli et pacis dc Grotius (1611) et le Leviathan de Hobbes (1651).

Cettc théorie du contrat devait avoir ses précurseurs en Allemagne avec Althusius (1557-1638) et Pubendorl (1632-1694).

Chacun foumil une anal yse specifique sur les modalités d accès à cette forme de pouvoir incarné dans l'État : pour Grotius, les individus se regroupaient sous l'effet d'une irtésistible appetilus socialis : chcz Hobbcs, la méchanceté des hommes lcs oblige à aliéner une partie de leurs libertés au profit d'une autorité supcricure : Althusius et Pubendorf deffendent, tous dcux, la vision d'un double contrat, l'un pour se constitucr en socicté, l'autre - de nivcau supérieur - pour former un Etat.

Voir à ce sujch. M. Abéles, Anthropologic de l'Etat, A. Colin, 1990, p. 13 et suivantes.

Herder mais aussi Humboldt insistent souvent sur la qualite anthropologique supericure des liens effecuffs ou affectifs. privilégiant l'attachement au "cocur, [à] l'ardeur, [au] sang, [à] l'humanité, [à] la vie". J.G. Herder. Une autre philosoplue de l'histoire, ouv. cité, p. 251.

53. La perception de la langue hasque en tant qu'instrument de ta raison modenc est en effet, comme on le verra plus loin, une nouveauté dans l"histoire des discours sur le basque.

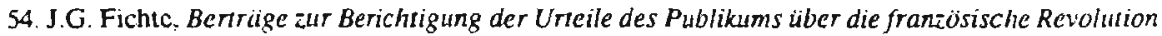
(1793), traduction françaisc. Considerations destinées à rectifier les jugements du public sur la Révolution Française. traduction de J. Berni, Payot, Paris, 1974.

56. Voir Aug. Rehberg, Untersuchungen Über die Franż̈sische Révolution (Recherches sur la Revolution Française. 1793). Pour celui-ci comme pour le précedent, le droit naturel se fonde sur la solidarite et I' accumulation intergénérationelle formant la tradition. selon une croissance organique expliquant la basc de constitution de chaque peuple. Voir G. Raulet, ouvrage cité, p. 298 ; voir aussi J. Droz. L'Allemagne et la Révolution Française. PUF, 1949. 1923

57. Voir J.G. Fichte, Discours à la Nation allemande, trad. franc. de J. Molitor, Ed. A. Costes, Paris,

\section{J.G. Fichtc. Considérations destinées..., ouv. citê, p. 294.}

59. La vision philosophique de l'histoire de l'Humanité comporte chez Fichte, cing grandes périodes ainsi caractérisécs : celle de l'Innocence où la raison gouveme sous la forme de l'instinct : celle du péché. où ce gouvernement s'excrce par une autorité extéricurc exigeant une obeissance aveugle ; celle du péché parfait. où se déchaine l'individualisme égoíste, indifférent à la vérité, et méprisant l'instinct, la raison, I'auturite. L homme se complaît en sa bassesse naturclle, dans le péché ; la période de justification, où la Raison et les kis sont comprises, la Vérité révérée, la Justice souveraine; celle de la justification absolue. de la sanctification ou de la liberté parfaite s'épanouissant en sainteté. Voir J.G. Fichte, Discours sur la Nation allemande, ouvragc cité. p. Xl et p. XII. Le XVIII“ siècle appartient à la troisième période d'où l'urgentc nécessite du rclèvement auquel tendent les Discours de Fichte.

60 . "Dans unc languc toujours restce vivante. cette partic suprasensible est symbolique; à chaque pas. elle synthctise, en une unité parfaite. l'enscmble de la vie nationale déposce dans la langue ; elle désignc aussi non pas unc idéc conventionnclle. mais une idée découlant nécessairement de toule la vie antérieurc de la nation : el de cette idéc et de cette désignation un ocil exercé dégagerait par une régression bien conduite l'histoire cntière du développement de la nation", écrit J.G. Fichte, ibidem, p. 65.

61. Ibidem. p. XIV.

62. Voir W.V. Humboldt. Introduction a l'aunre sur le Kavi el autres essais, trad. et introduction de P. Caussat, Lc Seuil, 1974.

63. J. Quillien, L'anthropologie philosophique de G. de Humboldt. Presses Universitaires de Lille. 1991. $664 \mathrm{p}$.

64. G. de Humboldt. La tache de l'historien. introduction de J. Quillien, traduction et notes d'A. Disselkamp cl A. Laks. Presses Universitaires de Lille, 1985.

65. Autcur notamment de Cosmos. Essais d une description physique du nonde et Voyage aux régions équinovales du noureau continem.

66. L'enscmble de son ocuvre a été l'objet d'unc grande édition réaliséc par l'Académie de Berlin et intitulke Gercmmehe Schrifien. B. Behr. 1903-1936 (l'cnscmble comprend 17 volumes répartis en 4 secLions: 1-Werke : 2-Politische Denkschriften : 3-Tagebuicher ; 4-Politische Briefe). 
La section Werke comprend. d'après J. Quillien : les textes théoriques (tomes 1 à 7. publiés de 1903 à 1907), les traductions (tome 9, 1908), les poc̀mes (tome 9, 1912) ct des suppléments dont un texte de 190 pages, perdu puis retrouvé, relatif au Pays Basque en 1801 (tome 13). Cette première édition réalisée à partir du principe chronologique a été suivie d'une autre construite autour de quelques regroupements thematiques: Werke in Fiinf Bäuden, Rg. Von Andreas Flitner Und KJaus Giel, Darmstadt, Wissemschaftliche Buchgescllschaft, 1960-1981

1 - Schriften zur Anthropologie und Geschichte, 1960.

2 - Schriften zur alterumskunde und Ästhetik. Die Vasken. 1961.

3 - Schriften zur Sprachphilosophic, 1963.

4 - Schriften zur Politik und zun Bildungswesen, 1984.

5 - Kleinc Schriften Autobiographisches. Dichtungen. Briefe, 1981.

67. Ainsi Essai sur les langues du Vieux Continent (1812), fragment d'une dissertation sur les langues nord-américaines destinées à l'ouvrage de son frère. Voyage aux régions équinoxales du nouveau continent. Relation historique. 2 volumes, édit. C. Minguet, Paris. Maspéro, 1980 ; Lettre d Monsieur Abel Rémusat sur la nature des formes grammaticales en général, et sur le génie de la langue chinoise en particulier. Paris, Lib. Orientale de Dondey-Dupré, 1827 ; "Mémoire sur la séparation des mots dans les textes sanscrits", dans Journal asiatique, 11, 1827, pp. 163-175 ; "Leture à Monsieur Jacquet sur les alphabets de la Polynésic asiatique" dans Nouveau joumal asiatique, 9,1832 , pp. 484-508. Il faut ajouter les traductions : Rome, poème traduit par G. Schweighäusser, Magasin Encyclopédique, IV, 1808, pp. 118-133; De l'origine des formes grammaticales et de leur infuence sur le développement des idées, trad. Alfred Tonnelli, suivie "de lanalise de l'opuscule sur la diversité dans la constitution des langues" Paris. A. Franck, 1859 ; Essai sur les limites de laction de l'Elat (1752), trad. et étude de Henri Chrétien, Paris, Germer-Baillière, 1867 : Recherche sur les habitanis primitifs de l'Espagne d l'aide de la langue basque (1820-1821), trad. A. Marrast. Paris, A. Franck, 1886 ; Introduction à l'auvre sur le Kavi et autres essais, trad. P. Caussat, Paris; Seuil, 1974 ('ouvrage comprend comme "autres essais" : "La tâche de l'historien" (pp. 33-63), "La recherche linguistique comparative" (pp. 65-96), "Le duel" (pp. 97-131) ; La 1Ache de l'hisiorien, ouvrage déjà cité.

68. Michel Foucault dans Les nots et les choses (Gallimard, 1966) analyse la structuration au début du $\mathrm{XIX}^{\mathrm{c}}$ siècle, du nouveau champ épistćmologique représenté par la linguistique, à travers les œuvres dc F. Schlegcl (La langue et la sagesse des Indiens, 1808), J. Gnmm (Deutsche Grammatik, 1818) et de F. Bopp (Système de comparaison du sanscrit. 1816) ct avec une très brève référence à Humboldt (p. 303), à propos de scs concepts d'argon el d'energeia appliqués à la langue : voir J. Quillien, ouvrage cité, p. 30.

La formation de tout nouvcau champ de savoirs pose les questions de la mise en évidence des transformations intcrvcnues dans l'épistémiè de la culturc et de niveau de conscience des personnes qui accomplissent ce travail de renouvellement des savoirs (sachant que les transformations dans l'épistémé se réalisent la plupart du temps a l'insu des chercheurs).

69. Lcturc à Schiller, 18 mai 1802, Ed. Seidel, tomme III, p. 221, cité par J. Quillien, ouvrage déjà citć, p. $600-601$.

70. G. de Humboldt. Fragmente der Monographie über die Basken. Gesammelte Schriften, 1903-1920, 1. 7. p. 598. cilte par J. Quillien, ouvrage cité, p. 601.

71. G. de Humboltd. Introduction d l'cetwre sur le Kavi, p. 71.

72. Voir J. Quillicn. ouvrage cite, p. 602.

73. Voir J. Michelet, Histoire de France, (1833-1846 et 1855-1867), T. I. pp. 437-447.

74. G. de Humboldt. Recherches sur les habilanis primitifs de l' Espagne dl ' aide de la langue basque. traduit de l'allemand par M. A. Marrast, Paris, Lib. A. Franck, 1866.

75. "Un noble et savant Prussien, appele de Humboldı se consacra à Paris à la lecure du dictionnaire basquc (...). Il venait délégué par quclque société de savants qui essaient de découvrir les langucs matrices, primitives et originales. Il va publicr bientôt quelque oeuvre sur cette langue méprisée pour beaucoup de savants espagnols qui... Le savant prussien vcut démontrer que la languc basque est éloquente. pure ct fécondc". cité par M. Marrast dans G. de Humboldt, Recherches sur les habitants primitifs de l'Espagne d̀ l'aide de la langue basque. ouvrage cité, p. VIII et LX.

76. Ibidem, p. VIII et IX.

77. Ibidem, ouv. cite. p. 7 .

78. Idcm.

79. Ibiden. p. 10. 
80. Ibidem. p. 15

81. Idem.

82. Idcm.

83. Ibidem, p. 72

84. Ibidem, p. 112.

85. Ibidem, p. 99.

86. Ibidem, p. 120.

87. Ibidem, p. 132.

88. Ibidem, p. 145.

89. Ibidem, p. 149.

90. 'Un individu singulier n'est par rapport à sa nation un individu qu'à la façon dont une feuille l'cst par rappor à un arbrc : parcillement les degrés d'individualité pcuvent s'étendre au-delà de la nation jusqu'à la souche ethnique, de cette dernierc jusqu'à la race, de celle-ci jusqu'à l'espèce humainc. Ce n'est qu'a l'intéricur d'un certain cercie que le terme subordonné peut alors progresser, régresser, ou différer" G. de Humboldt, La tâche de l'Historien, ouvrage déja cite, p. 49 ("Considerations sur l'histoire mondiale").

91. Ibidem, p. 47.

92. Idcm.

93. Humboldt était contemporain et ami de Friedrich August Wolf (1759-1824) considéré comme le fondatcur de la science de l'Antiquité à travers une philologic associant une pluralité de savoirs représentés par la grammaire, la géographie, l'hermémeutiquc, l'histoire des sciences el techniques, et appliquéc à Rome et à la Grèce.

94. "Dcs exemples de productions de force, de phénomènes que les circonstances ambiantes ne suffisent pas à expliquer, sont fournis par lc surgissement de l'art dans sa forme pure en Egypte que nous avons mentionnć ci-dessus, et peut-être plus encore par le développement subit en Grèce, de l'individualité libre cl cependant contenue dans des bornes définies : avec clle, la langue, la poésie et l'art atteignent d'emblée une perfection telle qu'il est vain d'en chercher le devenir progressif (...). La Grece offre unc ldee de l'individualité nationalc qui n'avait jamais existé auparavant, ct n'a plus jamais existé depuis, et de même que lc mystère de l'existence réside tout enticr dans l'individualité, de même tout progrès dans l'humanité. la perspective de l'histoire mondiale repose sur l'intensité, la liberté et la singularité de leur action réciproque", G. de Humboltdt, La tâche de l"historien". ouvr. déjà cité, p. 83.

95. "Il existe un moment de procréation moralc, où l"individu (nation, ou personne singulièrc) devient cc qu'il doit être. non par degres. mais soudainement cl tout d'un coup. Ce n'est qu'alors qu'il commence a ĉtre. car auparavant il élait un autre. Or ce débul est aussi sa perfection ; à parir de là, il régresse immédiatement. développant simpicment cc qui existe dćjà, et avec des forces qui declinent. Mais entre la conscience véritable du sommct et le moment où le déclin devient visible, il y a un moment d'hésitation, ct ćcst là la plus belle période", ibidem, p. 49.

96. "Corrcctions et rajouts pour la premicre partic du deuxième livre des Mithridates sur la langue basque ou cantabre". Berlin. 1817,93 p.

97. Mithridate ou Tableau Universel des Langues avec le Pater comme exemple, en cinq cents langucs ou idiomes. Bcrlin 1806-1815.

Cel ouvrage connut en Allemagnc. dès sa publication. un grand succès. Outre la traduction du Pater, on y trouve une histoire et une grammaire comparec portant sur un grand nombre de langues. Le premicr tome, qui aborde les langues asiatiques. est l'ocuvrc de J.C. Adelunez. Les quatre autres tomes rédigés d apres Ies memoires foumis par les frères Humboltd portent la signature de J.S. Vater.

98. Ibidem, p. 8.

99. Ibidem, p. 35.

100. Ibidem, p. 36 .

101. Idcm

102. Ibidem. P. 83 .

103. Ibidem. P. 84

104. G. de Humboldt. dans Gesammelte Schriften. Berlin. B. Berhr. 1904-1920 : traduit en espagnol. Los Vascos. Introducción y prólogo por Migucl de Unamuno. Ediciones Vascas, Bilbav, 1979. 
105. Ibidem, p. $9 \mathrm{cl} 10$.

106. Ibidem, p. 11 .

107. Idem.

108. Idem. "En csta su posición gcográfica se ha buscar tambien la clave de su historia toda, y en particular de la más remota".

109. Ibidem. p. 14.

110. Idem.

111. Idem.

112. Ibidem. p. 15.

113. Idem.

114. Idcm.

115. Idem.

116. Idem.

117. idem.

118. "A los vascus caractcriza idioma, organización, costumbres, fisonomia, y todo lo que le rndea, sin exceptuar cl aspecio de su pais. coma una estirpe pura y separada", ibidem, p. 18.

$119.1 \mathrm{dcm}$.

120. Idem.

121. Ibidcm. p. 19.

I22. Idcm.

123. Idem.

124. ldcm.

125. Ihidem. p. 21 .

126. Ihidcm. p. 22. L'approche anthropologique d'Humboldt. qui intègre bien des considérations herderiennes. sc retrouve dans l'œeuvre de l'anthropologue basque J.M. de Barandiaran lorsque celui-ci met en avanı le détcrminisme du "monde cxtéricur" (géographie, climat, ctc.) sur le "monde intérieur" et la congrucnce de ces deux mondes dans la formation des traits culturels. Quant à la languc, "la lengua - el cuskera - es clemento exiranrdinariamente significativo de la cultura vasca, no solo por ser expresión y vchiculo de ideas y sentimientos. sino también porque es portador de hucllas de diferentes cuituras y eco de numcrosas tradicioncs y de recuerdos historico-geograficos de Vasconia" "La langue - l'euskera - est un élément extraordinairement significati $\int \mathrm{de} \mathrm{la} \mathrm{culture} \mathrm{basquc.} \mathrm{non} \mathrm{sculement} \mathrm{en} \mathrm{étant} \mathrm{expression} \mathrm{ct} \mathrm{véhicule} \mathrm{des} \mathrm{idées}$ et des sentiments mais encore parce qu' clle est porteuse de traces de différcntes cultures a fait écho des nombrcuses traditions et des souvenirs historico-géographiques de Vasconie"), vnir J.M. Barandiaran, "Rasgos de la mentalidad popular Vasca" dans Primera Semana internacional de anthropología vasca (1970). Editorial La Gran Enciclopedia Vasca. Bilbao, 1971, pp 89.99 el en particulier la page 95.

\section{Ibidem}

128. Ibidem. p. 89.

129. Ibidem. p. 139.

130. "En estas viviendas aisladas nutre el vasco $\mathrm{cl}$ espírilu de libertad $\mathrm{c}$ independencia, que le distingue no cstando en cllas rodcado de nada cxurcho, se aficiona con apasionado amor a las peculiaridades de su mancra de vivir. de su nación y de su idioma : la pequeña hereda. en que con fatiga gana cl sustento de la fanilia, dcsarrolla la fucrza, la montaña que habita. le agilidad de sus miembros, y asi gana su estatura y su fisonomia cl scllo de vigor y denuedo en que se le reconoce a la primera mirada". íbidem, p. 140.

131. "L'esprit national est une conséquence du sentiment populaire, se limite avant tout au cercle qui critourc immédiatement l' homme. se caractérise donc toujours, simultanément, par l'intégration du proche et la marginalisation de l'ctranger. (...)". Ibidem, p. 81 .

132. Ibidem. p. 143.

133. Ihidem. p. 146.

134. "Aứn más imp̣orantes $\mathrm{e}$ interesantes aparccen estas moradas campestres aisladas, si se piensa en su influcncia sobre cl país y sobre cl caracter popular. Es incontestable que cl pais vasco tiene superioridades sobrc las restantes provincias españolas. que los vascongados son por lo menos tanto como todos los otros cspañoles en actividad. asiduidad. y que en ilustración ponpular. $\mathrm{en}$ verdadero patriotismo y genuino 
orgullo nacionał ninguna provincia se pueda igualar a las vascongadas. Aunque esto dependa tambićn a la vez de otras circunstancias. contribuyc empero cienamente por la mayor parte la ahora descrita distribución del país el gencro de vida de la parce agricultora de la nación", idem.

135. Ibidem. p. 149.

136. Ibidem, p. 149.

137. lbidem. p. 151. Celie-ci se remarque non pas tant dans les pas que dans les postures et les rotations du corps, Ics évolutions des gitanes d'Andalousic illustrant le mieux les débordements de la sensualitć.

138. Idem.

1.39. Ibidem, p. 152.

140. Idcm.

141. Idcm.

142. $\mathrm{ldcm}$.

143. Ibidem. p. 154.

144. Dans son ouvrage sur les danses basques et bcamaises. J.M. Guilcher évoque la dimension rituclle sociale très codifiée des conditions de pratique des danses selon lesquelles "la danse solennelle d'nuverture dc la fête [est] conduitc par le premicr magistral" et "les participants prennent place dans la chainc par rang ¿'ancienncté des maisons". Voir J.M. Guilcher, La tradition de danse en Bćarn el en Pawis Basque francais, Paris. Ed. Je la Maison dcs Scicnces de l'Homme, p. 46 et suivantes.

145. "En el hecho de que en cl país vascongado no sea propriamente popular el que cosas (como danzas y diversiones). que cn otras partes quedan abandonadas a la inclinación privada de cada uno. allí cn cicrto modo se hacen parte de la organización del país, estan bajo la inspección pública, y tienen una forma fija consuetudinaria genuinamente patria. y hasta diferente según el lugar de origen de cada una estriba notoriamcnte cn su mayor parte lo que en cl carácter del vascongado se clogia de preferencia sobre otras naciones. Afianza los lazos, que le ligan a su país y a sus conciudadanos, y nada puede reemplazar a la firmcza de estos lazos en la bienhcchora influencia sobre el vigor y la honrada rectitud del carácter. Aun la cultura mas clevada solo de un modo incompleto hace sus veces y nunca puede pasar en si misma todos los micmbros de una nación. pues, al revés, el amor a la patria y la ambición nacional toman aspectos en el mendigo y en los primeros del pucblo. En verdad que es natural, que con las crecientes relaciones con cl cxterior estas organizaciones siempre caen más en olvido ; con todo es de lamentar que la superioridad misma no valc mas por su conscrvación. De continuo se va adormeciendo una costumbre publica uras otra", Ibidem. p. 156 el p. 157.

146. Ibidem. p. 156.

147. "Así había en otro tjempo una solemnidad muy bencfica para la intcgridad de las costumbres (...)", Ibidem. p. 157.

148. Ibidem. p. 183. p. 182

149. "Ningun pucblo llcva tan lejos quizas la pasión por los cucntos como los vascongados". Ibidcm.

150. Idcm. Ce terme désigne un "esprit" qui trouble la vie domestiquc. Migucl de Unamuno. Ic traductcur de l'ouvrage, fait appel au terme basque d'iratxo pour désigner ce type d'esprit. Le mot de mamu pourrait également convenir.

151. Ibidem. p. 184.

152. Ibidem, p. 209.

$153.1 \mathrm{dcm}$.

154. Ibidem, p. 210.

155. lbidem. p. 211.

156. Ibidem. p. 210.

157. Idcm.

158. Idem.

159. Ibidem. p. 213.

160. Jden.

161. Idem. 
162. Ibidem, P. 213 et 214

163. Jbidem, p. 215.

164. Ibidem. p. 226.

$11 \mathrm{n}$ "accordc que deux lignes à la pratique de I'improvisation qui ne semble pas l'avoir frappt.

165. Idcm.

166. C'cst en 1859 que Moritz Lazarus a Heyman Stcinthal fondent la Zeitschrift fur Vökerpsychologie und spracimissenschaft ("Rcvue de psychologie et de linguistique des peuples"), entendant par le terme de "psychologic des pcuples", "une science de l"âme populaire", et plus exactement, selon la définition proposce par Steinthal lui-même, "une théorie des ćléments et des lois qui gouvement la vie spirituclle des pcuples". Voir à ce sujct, H. Bausinger, ouv. cité, p. $48 \mathrm{ct}$ suivantes.

167. Ibidcm, p. 227.

168. G. Bossong: "Wilhelm Von Humboldt y Hugo Schuchardt : dos eminentes vascologos alemanes" dans Arbor. Madrid, 1984. tomo CXLX, pp. 163-182, p. 167 et suivantes ; voir aussi G. Lacombe, "Hugo Schuchardt ct la morphologie de la Iangue basque", dans Revue Internationale des Etudes Basques. $\mathrm{t}$. XXVI. janvicr-mars, 1935. pp. 174-184.

169. D. Decamp. "The study of pidgin and creole languages". dans Dell Hymes (ed.), Pidginization and créolizuion of Languages, Cambridge University Press, 1971, pp. 13-39. p. 31.

Il cst a noter qu'un autre bascologue éminent Julien Vinson a consacré plusieurs études au domainc crécole à Ja demande de Schuchardt Jui-même : Dictionnaire des sciences anthropologiques. 1879. art. "créoles" : "compte rendu de Baissac : étude sur le patois crélc mauricien" dans Revue de linguistique et de philologie comparé. 1881. 14, pp. 415-420 ; "Un Échantillon du patois-français du Mozambique" dans Revue de linguistique et de philologie comparée. 1882, 15, pp. 330-332 : "Le conte du chat botté en patois créole de l'lle de la Réunion", trad. par E. Trouette, note introductrice, dans Revue de linguistique ef de philologie comparce, 1883.16. pp. 64-71.

170. Voir à cc sujet, L. Michelina. "L'euskaro-caucasien" dans A. Martincl (éd.), Le langage (Encyclopédic de la Plciade). 1968, pp. 1414-1437.

171. H. Schuchard. "Baskiche Studien. I : Über die Entstebung der Bezugsformen des Baskisehen Zcitwort", Denkschrifien der wiener Akadenie, 42, III, pp. 1-82.

172. Voir à ce propos. I. New-Altcmhcimer et D. Baggioni. "Recherches universitaires et idcolngics glottopolitiques en Catalogne ct en pays créolophones (Océan indien) à l'époque des néogrammairiens. 1880-1891" daris Langues. Revue de sociolinguistique. Montpellier, 1986, $\mathrm{n}^{\circ}$ 19. pp. 63-99, p. $65 \mathrm{cl}$ suivantes.

173. H. Schuchardi "Slawo-dcutsches und Slavo-italienisches" (Dem Herm von Miklosisch Zum 20 nov: 1883). Graz. pp. 3-140.

A cettc ćtude sur la sprachmischung dans l'Empirc austro-hongrois. il faut ajouter ces nombreux tra. vaux sur ic créclc dont : "Sur lc créole de la REunion" dans Romania, 1882, 11, pp. 589-593 : "Krcolische Studicn”. dans Sit-ungsiberichte der wiener Akademie der Wiss-PhiloHistorikerklasse. 1882-1890. 101. 1. II : 103, I : 104. II. 6. I : 122. IX.

174. Voir à ec propos 1. New-Altenheimer el D. Baggioni. article dejà cité, p. 96 ; D. Baggioni. "Gcographic linguistique et dialectologie dans le débat autour des lois phonétiques". dans Acter du $\lambda \mathrm{V} / \mathrm{l}$ Congres international de linguistique el philologie romanes, Aix en Provence. $1983:$ D. Bagguni. "Schuchardl 1 "incompris, ou du bon usage de la mixite des langues". dans Travaux de l"llA de la Réunion. 1984. n4, 24 p.; H. Schuchardt. Uber die Lanigesetze. Gegen die Junggrammaniker, Berlin, 1885.

175. Voir G. Lacombc. article dejà cité. p. 180.

Celui-ci ajoute : "Dans la mčme leture à Dodgson il déclare aussi s"être forcé đ"arriver à la clanté cn ajoutant que quelque chose peut êtư absolument clair et en même temps tuès difficile à comprendre. Il faut reconnaitre que ce livre n'a guère eté compris ni méme lu. mais enfin on ne doit pas déscspérer ct peut-čure dans l'avcnir sc trouvera-i-il quclque professcur d'Université qui consacrera une série de leçons a un exp̧k méthodique ct prectis' de ce que Schuchardı a pensé du verbe basque. Ccla consolera peut-être Schuchardt par dela la tombe de l'amertume d'ailleurs toutc relative qu'il dut eprouver à constater que divers bascologucs $n$ apprćcierent pas son travail. Vinson lui consacra un article auquel Schuchardı dut répondre prour en corriger les crreurs d"intcrprétation. Webster fit tgalement à son sujet des contresens que Schucharut relcva : Dodgson fut cmbarrasse et Van Eys bien qu ayant couvert dannotations son excmplairc de cct ouvragc. hicn qu'ayant pris par ailleurs plusieurs pages de notes et même cortespondu avec Schuchardt. Iit dans la rcvuc anglaisc The Academ: une recension brève el peu compromettante et dans unc lcttre de 1895. il déclare que les théorics de Schuchardt bouleversent des notions reçues. qu'il faut bien peser le pour ct le contre, qu il ne sait que decider eı qu une difficulté pour ainsi dire psychologique l'arrête". idem. 
176. Voir le numero 2 de la revuc Euskara, Berlin, 1886, p. 13.

Les statuts précisent ainsi la position du sociétaire : "Peut devenir membre de la société toute personne qui s'intćressc à la langue basque ct à sa vulgarisation. notamment tout énudit, philologue etc., s'occupant de cettc langue ou desirant cn faire l'nbjet de ses éludes", jdem.

Quant à l'organc de la societté. clle doit répondre aux objectifs suivants : "Afin de présenter utilcment les intérêts de la société dans toutes les directions et pour l'aider à atteindre le but qu'elle se propose. la création d'un organe proprc à la société est désirable. Les débours résultants pour elle d'une semblable cnureprise scront couverts au moyen des cotisations des societaires. Ceue publication se nommera Euskara, paraîtra provisoirement sans être tenuc à des époques fixes, ct comprendra une feuille en ${ }^{\circ}$; plus tard, lorsque lencaisse lc permettra, clle aura de une à deux fcuilles de textc el deviendra trimestrielle. Elle sera rédigéc cn langue alicmande, sans exclure les articles écrits en basque ou en toute autre langue propre à scrvir les intérêts de la "Baskischen Gesellschafi" et de féconder son travail, comme par exemple les langues francaisc cl espagnole.

Lc droit de collaboration appartient à tout sociétaire, mais les articles destinés à l'insertion devront être ccrits en allemand, ou dans un des idiomes déjà indiques. Ils ne pourront traiter que de la langue basque. de l'histoire du pcuplc et du Pays Basque, de la question ibcricnne, etc.". Ibidem, p. 14.

Le même numéro informe égalemcnt que le dcuxième secrêtaire de la société est V. Stempr, rêsidant à Bordcaux. autcur notamment de Besital die baskische Sprache ein transitives Zeitwort, oder nicht?, Bordcaux. 1890, 16 p. ; et en français, La languc basque possede-t-elle. oui ou non, un verbe transitif?. traduit de l'allemand avec quelques modifications, Bordcaux. 1890, $15 \mathrm{p}$. 